\title{
Introducing a variable speed of sound in single-component Lattice Boltzmann simulations of isothermal fluid flows
}

\author{
N. Looije \\ Department of Chemical Engineering, Delft University of Technology, the Netherlands \\ J.J.J. Gillissen \\ Department of Mathematics, University College London, Gower Street, London WC1E \\ 6BT, United Kingdom \\ S. Sundaresan
}

Department of Chemical and Biological Engineering, Princeton University, USA

H.E.A. Van den Akker*

Bernal Institute, School of Engineering, University of Limerick, Ireland

\begin{abstract}
To simulate the hydrodynamics and mixing characteristics of chemical reactors by means of a lattice Boltzmann method (LBM), it is essential to consider components with varying molecular weights (and therefore speeds of sound). This option requires modification of the standard equilibrium distribution function and the use of an extended velocity set. In this paper, we show that, for isothermal incompressible single-component non-reactive flows, tuning the speed of sound with a modified equilibrium distribution and an extended velocity set allows for reproducing the proper flow characteristics with strongly reduced errors compared to LBM simulations on standard lattices. This is done for two isothermal benchmarks, viz. a damped standing pressure wave and a decaying viscous Taylor-Green vortex. The convergence as a function of the number of lattice nodes used improves substantially for varying values of the speed of sound.
\end{abstract}

Keywords: lattice Boltzmann methods, incompressible, extended velocity set,

\footnotetext{
${ }^{*}$ Corresponding author

Email address: harry.vandenakker@ul.ie (H.E.A. Van den Akker)
} 
tunable speed of sound, damped pressure wave, Taylor-Green Vortex

\section{Introduction}

This paper reports on our results with respect to introducing, exploiting and assessing variable speeds of sound in isothermal lattice Boltzmann (LBM) flow simulations. Our wish to introduce variable speeds of sound stems from our interest in computationally simulating chemical reactors operated either in batch, semi-batch, or continuous flow modes. A rather unexplored aspect of simulating reactive flows by means of lattice Boltzmann methods (LBMs), however, relates to the differences in molecular mass that are typical of chemical reactions where mass is conserved but the number of moles and molecular mass vary between reactants and products [1, 2]. One of the options for dealing with these variations in molecular mass is by means of a tunable speed of sound.

In LBM, the speed of sound $c_{s}$ plays a major role although it is not a physically meaningful property of an incompressible fluid [3. It figures predominantly in the Chapman-Enskog multi-scale analysis that relates the (discretized) Boltzmann equation to the macroscopic continuum description [4-6]. In the LBM approach, (partial) pressure, temperature and kinematic viscosity are all proportional to $c_{s}^{2}$, as in an ideal gas. In general, we have the isothermal sound speed $c_{s}^{2}=\frac{R T}{M}$, where $R$ is the universal gas constant, $T$ is temperature, and $M$ is the molecular mass. This follows from the ideal equation of state $p=\frac{\rho R T}{M}$ and $c_{s}^{2}=\left.\frac{\partial p}{\partial \rho}\right|_{T}$. In the standard isothermal LBM, the speed of sound is fixed and determined by the lattice. For certain applications, however, such as the chemical reactors of our interest, it is necessary to simulate components with different molecular masses and therefore different sound speeds. Specifically, we require a component $\sigma$ to have different equations of state $p_{\sigma}=\frac{\rho_{\sigma} R T}{M_{\sigma}}=\rho_{\sigma} c_{s, \sigma}^{2}$ and the total pressure $p=\sum_{\sigma} p_{\sigma}$. For such systems, we must be able to tune the speeds of sound of the components while retaining a correct description of the hydrodynamics.

With eventual applications to flows in chemical reactors in mind, the purpose of this paper is to demonstrate the feasibility of retaining correct hydrodynamics while using a variable isothermal sound speed. The meso-scale LBM is known to be excellently capable of simultaneously dealing with thermodynamic, hydro- 
dynamic and chemical phenomena and processes in a computationally efficient and elegant way. It is also known, however, that in non-isothermal flows satisfying the constraints posed by the thermohydrodynamic equations requires the extension of the LBM velocity set beyond the minimum number that is used in the standard isothermal LBM [7-10]. Since in the LBM the speed of sound is proportional to temperature, it is clear from these studies that also under isothermal conditions tuning the speed of sound, while not necessarily spatially or temporally variant, requires the use of lattices with additional velocities to retain correct hydrodynamics.

In this paper, we first show we must both modify the equilibrium distibution and use an extended velocity to allow a variable speed of sound. For our present purpose, simulations of a single component system are sufficient to demonstrate the ability to vary a component's speed of sound, while, compared to multicomponent systems, such single-component simulations allow a more transparent analytical treatment (Chapman-Enskog), easier numerical implementation, and test cases with simpler boundary conditions compared to multi-component systems. Nonetheless, our eventual purpose is to exploit the combination of a tunable speed of sound, a modified equilibrium distribution and an extended velocity set for the purpose of simulating convective transport and mixing in both stirred and continuous-flow chemical reactors with multiple components. This application of the present work will be the topic of a future paper. Compressibility artifacts and acoustics topics are explicitly outside the scope of the present paper as they are irrelevant to the chemical reactors of interest and we consider only flows with low Mach numbers.

In general, to vary the speed of sound in isothermal LBMs, we can choose between two types of lattice implementations which may be denoted as offlattice and on-lattice propagation schemes, respectively. Alternative methods have also been investigated, e.g. it was shown by Buick \& Cosgrove [11 that a variable speed of sound may be introduced through a modified body force. However, within the scope of this work we will limit the discussion solely to lattice implementations.

For non-flowing systems, McCracken \& Abraham [12] exploited both offlattice and on-lattice schemes for simulating diffusive mass transfer in binary 
mixtures with varying molecular masses. Off-lattice schemes represent a multiple lattice approach where components, depending on their relative weights, travel only fractions of the lattice spacing in a lattice time step [12-14]. An off-lattice scheme was used by Arcidiacono et al. [13] to describe the behavior of several chemical species when simulating the oxidation of methane in a channel with catalytic walls. While physically sound, off-lattice schemes require computationally expensive interpolation steps and also complicate the boundary conditions required in many engineering applications.

Compared to off-lattice schemes, on-lattice schemes are more attractive since they require little modification in standard numerical codes and preserve the local nature of LBMs. The idea behind on-lattice propagation schemes is to manipulate the equilibrium moments in such a way as to properly recover the required hydrodynamic equations in the macroscopic limit.

Applied to standard velocity lattices, however, on-lattice schemes, as described in the literature [3, 5, 12, 15-19] suffer from an inherent flaw as they do introduce unphysical error terms into the Navier-Stokes equation (see Section II $\mathrm{B}$ and AppendixA. These error terms are the result of the limited number of velocities available in standard LBM lattices and render the scheme less accurate when applied to systems involving fluid flow. It can be shown that in 1D the error terms associated with varying the sound speed on standard LBM lattices take the form of a viscous stress and can be mitigated by rescaling the viscosity [3]. This, however, is not possible in higher dimensions, where a hydrodynamically correct modification of the sound speed requires a lattice with additional velocities.

Many of the above-cited researchers sticking to the standard velocity lattice were interested in acoustics and compressibility effects. For example, Alexander et al. [15] implemented fluid compressibility effects by modifying the equilibrium distribution without extending the velocity lattice. In spite of the resulting incorrect formulation of the hydrodynamics they used this approach while relaxing the low-Mach number restriction of the conventional LBM; this allowed them to simulate shock-wave formation through the viscous Burgers equation by using the LBM framework. As said, acoustics and compressible flows are beyond the scope of this paper. 
To resolve the issue of the unphysical error terms in the Navier-Stokes equation, more velocity directions must be added to the LBM lattice. We refer to these lattices, with an extended velocity set, as "extended velocity lattices". To the best of our knowledge, only a few researchers used an extended velocity lattice with the view to tune the isothermal speed of sound in LBM [7, 20]. Qian [7] proposed extended velocity sets along with modified equilibrium distributions for simulating 1D, 2D and 3D thermohydrodynamic cases - which can be simplified for isothermal conditions. In their paper on multiphase systems, Chai and Zhao [20, proposed a multiple-relaxation-time scheme to allow for the use of different speeds of sound for different components of different molecular mass. This effectively introduced a modified equilibrium distribution on a D2Q13 lattice, however this was done without further derivation or analysis. However, a general and systematic theoretical treatment and evaluation of the accuracy and applicability of a variable-speed-of-sound approach for isothermal single-phase flows has not yet been reported.

In the present paper, we will therefore first set the scene by showing once more that modifying the equilibrium distribution on a standard D1Q3 or D2Q9 lattice does not allow tuning of the speed of sound and that resolving this problem requires extending the velocity lattice, i.e. to D1Q5 or D2Q13. We will then introduce equilibrium distribution functions on these extended lattices in the BGK collision framework. While several early papers on (multi-component) LBM, such as [17], already mention the importance of a 'proper choice' of the equilibrium distribution in order to change the sound speed, most studies were conducted on standard velocity lattices and do not recover correct hydrodynamics.

We will investigate the accuracy of this novel approach for two canonical isothermal single-phase single-component flows and will find just marginal errors in the velocity and density fields. The novelty of our work is that we provide a systematic theoretical treatment, including a derivation that proves the necessity of using extended velocity lattices to extend the isothermal LBM sound speed and an evaluation of the accuracy and range of applicability of this approach. Furthermore, compared to Qian's as well as Chai and Zhao's papers, we use a different equilibrium distribution, and our validation focuses on 
flow field predictions while their validation focused on viscosities, (self-)diffusion coefficients and phase separation.

The remainder of this paper comprises of the following sections: Section II reviews the need for extended velocity lattices when changing the sound speed in isothermal LBMs. It also presents the newly proposed on-lattice propagation scheme using a modified equilibrium distribution. Then, the improved accuracy of our scheme is assessed with the help of two canonical cases of fluid flow; in Section III, we compare numerical simulations with the analytical solution for the case of a viscously damped standing pressure wave, while in Section IV we do the same for the Taylor-Green vortex.

\section{An alternative on-lattice propagation scheme}

\subsection{Conventional theory}

The kinetic LBM equation which governs the evolution of a mass distribution function $f_{i}$ is given by:

$$
\frac{f_{i}\left(x_{\alpha}+e_{i, \alpha} \triangle t, t+\triangle t\right)-f_{i}\left(x_{\alpha}, t\right)}{\triangle t}=\Omega_{i}\left(x_{\alpha}, t\right)
$$

where $i$ runs over the number of velocity directions $e_{i, \alpha}$, see Fig. 11. Greek indices are used to denote spatial directions; the Einstein summation convention is implied for repeated Greek indices. The left-hand side of Eq. 11 represents the propagation of particles from lattice site $x_{\alpha}$ at lattice time $t$ to $x_{\alpha}+e_{i, \alpha} \triangle t$ at lattice time $t+\triangle t$ whereas the right-hand side represents the collision of particles at lattice site $x_{\alpha}$ at lattice time $t$. For conventional lattices, $f_{i}$ propagates to nearest neighbours in $1 \mathrm{D}$ and to the nearest and next nearest neighbours in 2D, while for extended lattices, it also propagates to more extended sets of neighbours, see Fig. 1 .

The collision operator, $\Omega_{i}$, follows a conventional BGK relaxation process 21]:

$$
\Omega_{i}\left(x_{\alpha}, t\right)=-\frac{f_{i}\left(x_{\alpha}, t\right)-f_{i, e q}\left(x_{\alpha, t}\right)}{\tau}
$$

which models a simple relaxation, with relaxation time $\tau$, of the distribution function towards an equilibrium distribution function, $f_{i, e q}$. In conventional 

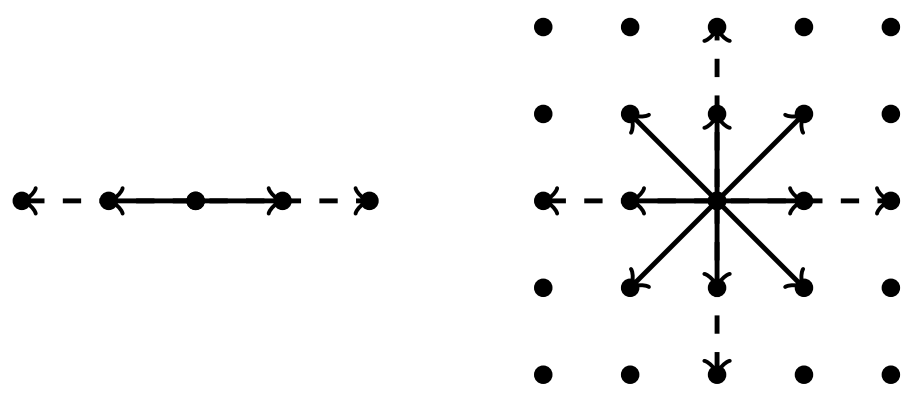

Figure 1: The lattice velocity set for D1Q3 (left) and D2Q9 (right) conventional lattices (solid lines) and D1Q5 (left) and D2Q13 (right) extended velocity lattice (dashed lines)

LBMs, this equilibrium distribution function is an expansion of the MaxwellBoltzmann distribution up to second-order terms in the Mach number:

$$
f_{i, e q}=w_{i} \rho\left[1+\frac{e_{i, \alpha} u_{\alpha}}{c_{s}^{2}}+\frac{1}{2} \frac{\left(e_{i, \alpha} e_{i, \beta}-c_{s}^{2} \delta_{\alpha \beta}\right) u_{\alpha} u_{\beta}}{c_{s}^{4}}\right]
$$

where $w_{i}$ and $c_{s}$ are the quadrature weights corresponding to a particular velocity direction and the lattice speed of sound respectively. From the mathematical treatment of Eq. [1 it is known that the weights and velocity vectors have to satisfy a specific set of symmetry conditions [22, 23, namely:

$$
\sum_{i} w_{i}=1 \quad \sum_{i} w_{i} e_{i, \alpha}=0 \quad \sum_{i} w_{i} e_{i, \alpha} e_{i, \beta}=c_{s}^{2} \delta_{\alpha \beta}
$$

where the second moment of the weights is used to define the lattice speed of sound, which, as a function of the chosen lattice, is a constant. The values of $w_{i}, e_{i, \alpha}$ and $c_{s}$ for the lattices used in this research are found in Table 1

The equilibrium distribution function in Eq. 3 is uniquely defined by the macroscopic density $\rho$ and velocity $u_{\alpha}$ which by definition are determined by the zeroth and first moments, respectively, of the distribution functions, $f_{i}$ :

$$
\rho=\sum_{i} f_{i} \quad \rho u_{\alpha}=\sum_{i} e_{i, \alpha} f_{i}
$$

Furthermore, the choice of the above form of Eq. 3 ensures also that:

$$
\rho=\sum_{i} f_{i, e q} \quad \rho u_{\alpha}=\sum_{i} e_{i, \alpha} f_{i, e q}
$$

which along with the second and third moments [6]: 


\begin{tabular}{|c|c|c|c|c|c|c|c|}
\hline lattice & \multicolumn{3}{|c|}{ weights, $w_{i}$} & \multicolumn{3}{|c|}{ velocity, $e_{i}$} & $\mathrm{ss}, c_{s}$ \\
\hline direction $i$ & 1 & $2 \rightarrow 3$ & $4 \rightarrow 5$ & 1 & $2 \rightarrow 3$ & $4 \rightarrow 5$ & \\
\hline D1Q3 & $\frac{2}{3}$ & $\frac{1}{6}$ & - & 0 & $\cos \left(\frac{\pi}{2}(i-1)\right)$ & - & $\frac{1}{\sqrt{3}}$ \\
\hline D1Q5 & $\frac{1}{2}$ & $\frac{1}{6}$ & $\frac{1}{12}$ & 0 & $\cos \left(\frac{\pi}{2}(i-1)\right)$ & $2 \cos \left(\frac{\pi}{2}(i-1)\right)$ & 1 \\
\hline
\end{tabular}

(a) one-dimensional lattices

\begin{tabular}{|c|c|c|c|c|c|c|c|c|}
\hline lattice & \multicolumn{5}{|c|}{ weights, $w_{i}$} & \multicolumn{2}{|c|}{ velocity, $\vec{e}_{i}$} \\
\hline \hline direction $i$ & 1 & $2 \rightarrow 4$ & $5 \rightarrow 8$ & $9 \rightarrow 13$ & 1 & $2 \rightarrow 4$ & $5 \rightarrow 8$ & $9 \rightarrow 13$ \\
\hline D2Q9 & $\frac{4}{9}$ & $\frac{1}{9}$ & $\frac{1}{36}$ & - & {$\left[\begin{array}{l}0 \\
0\end{array}\right]$} & {$\left[\begin{array}{c}\cos \left(\frac{\pi}{2}(i-1)\right) \\
\sin \left(\frac{\pi}{2}(i-1)\right)\end{array}\right]$} & $\sqrt{2}\left[\begin{array}{c}\cos \left(\frac{\pi}{2}(i-1)+\frac{\pi}{4}\right) \\
\sin \left(\frac{\pi}{2}(i-1)\right)\end{array}\right]$ & - \\
\hline D2Q13 & $\frac{3}{8}$ & $\frac{1}{12}$ & $\frac{1}{16}$ & $\frac{1}{96}$ & {$\left[\begin{array}{l}0 \\
0\end{array}\right]$} & {$\left[\begin{array}{l}\cos \left(\frac{\pi}{2}(i-1)\right) \\
\sin \left(\frac{\pi}{2}(i-1)\right)\end{array}\right]$} & $\sqrt{2}\left[\begin{array}{l}\cos \left(\frac{\pi}{2}(i-1)+\frac{\pi}{4}\right) \\
\sin \left(\frac{\pi}{2}(i-1)+\frac{\pi}{4}\right)\end{array}\right]$ & $2\left[\begin{array}{l}\cos \left(\frac{\pi}{2}(i-1)\right) \\
\sin \left(\frac{\pi}{2}(i-1)\right)\end{array}\right]$ \\
\hline
\end{tabular}

(b) two-dimensional lattices

Table 1: Quadrature weights, lattice velocity vectors and speed of sound (ss) for 1D and 2D lattices.

$$
\begin{gathered}
\sum_{i} e_{i, \alpha} e_{i, \beta} f_{i, e q}=\rho c_{s}^{2} \delta_{\alpha \beta}+\rho u_{\alpha} u_{\beta} \\
\sum_{i} e_{i, \alpha} e_{i, \beta} e_{i, \gamma} f_{i, e q}=\rho c_{s}^{2}\left(u_{\alpha} \delta_{\beta \gamma}+u_{\beta} \delta_{\alpha \gamma}+u_{\gamma} \delta_{\alpha \beta}\right)
\end{gathered}
$$

are required to resolve the proper macroscopic behaviour in the incompressible limit [5, 12 .

Using Eqs. 6-8 in a multiscale analysis (see AppendixA, the macroscopic equations which are modeled by Eq. 1 are the continuity equation and NavierStokes equations [4] 6 :

$$
\begin{gathered}
\partial_{t} \rho+\partial_{\alpha} \rho u_{\alpha}=0 \\
\partial_{t} \rho u_{\alpha}+\partial_{\beta} \pi_{\alpha \beta}=0
\end{gathered}
$$

where the momentum flux tensor, $\pi_{\alpha \beta}$, is defined as:

$$
\pi_{\alpha \beta}=\rho u_{\alpha} u_{\beta}+p \delta_{\alpha \beta}-\sigma_{\alpha \beta}
$$

Here, we identify the viscous stress tensor $\sigma_{\alpha \beta}$ :

$$
\sigma_{\alpha \beta}=\rho \nu\left(\partial_{\beta} u_{\alpha}+\partial_{\alpha} u_{\beta}\right)
$$

The stress tensor $\sigma_{\alpha, \beta}$ in Eq. 11 also contains a spurious error term of $O\left(u^{3}\right)$ (not shown here), which originates from the second-order Mach number 
expansion of the equilibrium distribution in Eq. 3 and effectively makes this scheme spatially 2nd order accurate. It must be noted that this spurious term can in principle be mitigated on extended velocity lattices by employing an $O\left(\mathrm{Ma}^{3}\right)$ expansion of the equilibrium distribibution [24, which extends the range of Mach numbers that can be simulated. In this work however we do not follow such an approach. Instead we propose to deal with different sound speeds by modifying Eq. 3, while retaining its basic polynomial form. Consequently the proposed method is restricted to small Mach numbers, which is suited for our field of application dealing with incompressible flows.

The pressure in Eq. 10 and the viscosity in Eq. 11 are found to depend on the speed of sound $c_{s}$ :

$$
p=\rho c_{s}^{2} \quad \nu=c_{s}^{2} \triangle t\left(\frac{\tau}{\triangle t}-\frac{1}{2}\right)
$$

This models a fluid flow with an ideal gas equation of state which conventionally has a proportionality constant inversely proportional to the square root of the molecular mass. In this model, as the speed of sound is a lattice-dependent constant and we are considering an isothermal flow, this implies that the molecular mass may not be varied. As it is our aim to allow the varying of the molecular mass, we need a model which is able to tune the speed of sound to our specification.

\subsection{The modified equilibrium distribution}

The aim is to find a model which allows a tunable speed of sound. As the macroscopic equations in Eq. 9 are defined in terms of the equilibrium moments Eqs. 6[8 we require new definitions of these moments to allow a variable speed of sound. We begin by proposing a new form for the equilibrium distribution:

$$
\tilde{f}_{i, e q}=w_{i} \rho\left[A_{i}+B_{i} \frac{e_{i, \alpha} u_{\alpha}}{c_{s}^{2}}+\frac{1}{2} \frac{\left(e_{i, \alpha} e_{i, \beta}-c_{s}^{2} \delta_{\alpha \beta}\right) u_{\alpha} u_{\beta}}{c_{s}^{4}}\right]
$$

where the velocity direction-dependent coefficients $A_{i}$ and $B_{i}$ are introduced as degrees of freedom in order to satisfy the equilibrium moments. Clearly, the conventional equilibrium distribution in Eq. 3 is recovered when $A_{i}=B_{i}=1$. It must be noted that a proper derivation of such an equilibrium distribution should be done through a Hermite expansion [24]. However the present approach, which is in line with other references [5, 12, 16, 17, 25], provides a 
straightforward and easily implementable method of varying the speed of sound once $A_{i}$ and $B_{i}$ are determined..

In order to have a variable speed of sound, we keep the zeroth and first equilibrium moments the same as in Eq. 6 but modify the second and third equilibrium moments in Eqs. 7 \& 8 to read:

$$
\begin{gathered}
\sum_{i} e_{i, \alpha} e_{i, \beta} \tilde{f}_{i, e q}=\rho c_{s, e}^{2} \delta_{\alpha \beta}+\rho u_{\alpha} u_{\beta} \\
\sum_{i} e_{i, \alpha} e_{i, \beta} e_{i, \gamma} \tilde{f}_{i, e q}=\rho c_{s, e}^{2}\left(u_{\alpha} \delta_{\beta \gamma}+u_{\beta} \delta_{\alpha \gamma}+u_{\gamma} \delta_{\alpha \beta}\right)
\end{gathered}
$$

where an effective speed of sound, $c_{s, e}$, is introduced which is the lattice speed of sound scaled with a parameter $\gamma$ :

$$
\gamma=\frac{c_{s, e}^{2}}{c_{s}^{2}}
$$

Note that the model reverts to the conventional model when $\gamma=1$.

With these redefined equilibrium moments the viscous stress tensor becomes:

$$
\sigma_{\alpha \beta}=\rho \nu_{e}\left(\partial_{\beta} u_{\alpha}+\partial_{\alpha} u_{\beta}\right)
$$

with a redefined pressure and viscosity:

$$
p=\rho c_{s, e}^{2} \quad \nu_{e}=c_{s, e}^{2} \triangle t\left(\frac{\tau}{\triangle t}-\frac{1}{2}\right)
$$

Comparing to Eq. 12, the pressure and viscosity have been redefined in terms of the effective speed of sound, $c_{s, e}$.

Satisfying the equilibrium moment relations in Eqs. $7 \& 8$ by using Eq. 13 requires a number of degrees of freedom in the equilibrium distribution functions in $1 \mathrm{D}, 2 \mathrm{D}$ or $3 \mathrm{D}$, respectively. The standard lattices D1Q3, D2Q9, D3Q15, D3Q19 and D3Q27 do not contain enough degrees of freedom for this purpose. These lattices only facilitate correct equilibrium moments when $c_{s, e}=c_{s}$, or $\gamma=1$, while for other values of the sound speed the moments can not be simultaneously satisfied. Therefore these standard lattices do not allow for a tunable sound speed by modifying only the equilibrium distribution function.

Notwithstanding this finding is not novel [23, several authors [5, 12] did tune the sound speed on standard lattices by satisfying moments up to rank two only 
(Eqs. 6 \& 7) while keeping the third moment (Eq. 15) at $\gamma=1$. A multiscale analysis (Appendix A), however, shows that introducing a tunable speed of sound and satisfying the equilibrium moments up to just rank two results in an error term in the viscous stress $\sigma_{\alpha, \beta}$ of the Navier-Stokes equations:

$$
\sigma_{\alpha \beta}=\rho \nu_{e}\left[\partial_{\alpha} u_{\beta}+\partial_{\beta} u_{\alpha}\right]+\varepsilon_{\alpha \beta}
$$

where the error term takes the form:

$$
\varepsilon_{\alpha \beta}=(1-\gamma) \nu\left[\partial_{\gamma}\left(\rho u_{\gamma}\right) \delta_{\alpha \beta}+\partial_{\alpha}\left(\rho u_{\beta}\right)+\partial_{\beta}\left(\rho u_{\alpha}\right)\right]
$$

where $\nu$ is the viscosity based on the lattice speed of sound. Eq. 20 shows that these terms are identically zero for two cases: (a) when the sum of all these velocity gradients is zero - a condition excluding virtually all flow conditions; (b) when $c_{s, e}=c_{s}$, i.e. $\gamma=1$. It is further noted that in the limit of small density perturbations $(\mathrm{Ma} \ll 1)$ the leading order contribution to the error term reads:

$$
\varepsilon_{\alpha \beta}=(1-\gamma) \rho \nu\left(\partial_{\gamma} u_{\gamma} \delta_{\alpha \beta}+\partial_{\alpha} u_{\beta}+\partial_{\beta} u_{\alpha}\right)
$$

In $1 \mathrm{D}$, this equation reduces to a viscous stress:

$$
\epsilon_{\alpha \beta}=3(1-\gamma) \rho \nu \partial_{x} u_{x}
$$

which shows that for $\mathrm{Ma} \ll 1$, the error term can be mitigated by a rescaling of the viscosity 3 :

$$
\nu_{e f f}=\nu_{e}+\frac{3}{2}(1-\gamma) \nu
$$

However, for higher dimensional problems, the error term cannot be mitigated in this way, resulting in non-physical hydrodynamic behaviour.

In conclusion, to model the correct macroscopic behaviour, one needs to satisfy equilibrium moments up to rank three using Eq. 13. The standard lattices can facilitate this only when the speed of sound equals $c_{s}$. For sound speeds other than $c_{s}$ however, the standard lattices are not sufficient and one needs to extend the number of velocity directions to properly satisfy the equilibrium moments up to rank three. 


\subsection{Evaluation of the coefficients}

The requirement of Eqs. 6 8 on the coefficients in Eq. 13 results in a system of equations for the coefficients $A_{i}$ :

$$
\begin{gathered}
\sum_{i} w_{i} A_{i}=1 \quad \sum_{i} w_{i} e_{i, \alpha} e_{i, \beta} A_{i}=c_{s, e}^{2} \delta_{\alpha \beta} \\
\sum_{i} w_{i} e_{i, \alpha} A_{i}=\sum_{i} w_{i} e_{i, \alpha} e_{i, \beta} e_{i, \gamma} A_{i}=0
\end{gathered}
$$

and the coefficients $B_{i}$ :

$$
\begin{gathered}
\sum_{i} w_{i} e_{i, \alpha} e_{i, \beta} B_{i}=c_{s}^{2} \delta_{\alpha \beta} \\
\sum_{i} w_{i} e_{i, \alpha} e_{i, \beta} e_{i, \gamma} e_{i, \delta} B_{i}=c_{s}^{2} c_{s, e}^{2} \triangle_{\alpha \beta \gamma \delta} \\
\sum_{i} w_{i} e_{i, \alpha} B_{i}=\sum_{i} w_{i} e_{i, \alpha} e_{i, \beta} e_{i, \gamma} B_{i}=0
\end{gathered}
$$

where $\triangle_{\alpha \beta \gamma \delta}=\delta_{\alpha \beta} \delta_{\gamma \delta}+\delta_{\alpha \gamma} \delta_{\beta \delta}+\delta_{\alpha \delta} \delta_{\beta \gamma}$.

In this system of equations, Eqs. $22 \& 25$ imply all odd moments of the coefficients to be identically zero which indicates that the odd-moment equations are linearly dependent. A further simplification is made by assuming the coefficients are subject to symmetry conditions where coefficients for lattice directions $i$ with equal lattice velocity magnitudes $\left|\vec{e}_{i}\right|$ are equivalent. As an example in D1Q3, $\left|\vec{e}_{2}\right|=\left|\vec{e}_{3}\right|$, therefore $A_{2}=A_{3}=A_{2 \rightarrow 3}$ and $B_{2}=B_{3}=B_{2 \rightarrow 3}$ where $A_{2 \rightarrow 3}$ and $B_{2 \rightarrow 3}$ is simply notation for the combination of coefficient $A_{2}$ and $A_{3}$ and $B_{2}$ and $B_{3}$; in D1Q5, the velocity set is extended to include directions where $\left|\vec{e}_{4}\right|=\left|\vec{e}_{5}\right|$ and there $A_{4}=A_{5}=A_{4 \rightarrow 5}$ and $B_{4}=B_{5}=B_{4 \rightarrow 5}$. This is easily extended to multiple dimensions. The assumption of these symmetry conditions on the coefficients automatically satisfies the odd moments and the anti-symmetric parts of the second moments of these coefficients. In addition, $B_{1}$ can be set to zero since the system of equations for coefficients $B_{i}$ is only defined in terms of moments larger than the zeroth moment. A result of this is that for single-speed lattices, i.e. D1Q3 or D2Q9, a variable speed of sound is not possible as there are insufficient coefficients (see AppendixB.1). Instead, a multi-speed lattice, i.e. D1Q5 or D2Q13, must be used (see AppendixB.2. 
The above simplifications result in a underspecified system of equations requiring additional relation in terms of the coefficients. Following the approach in the literature [5, 12], these relations are chosen in the form $A_{i}=r A_{j \neq i}$ and $B_{i}=r B_{j \neq i}$ where $r$ is a degree of freedom used to tune the speed of sound. Depending on the dimensionality of the problem, there may be different sets of relations which close the system of equations and yield proper solutions. In this case, we chose a set which yields the simplest and most physically sound set of solutions; it differs from the sets chosen by Chai and Zhao [20] and Qian 7 .

As shown in AppendixB.2 our choice leads to the following expressions for the coefficients $A_{i}$ and $B_{i}$ for D1Q5:

$$
\begin{gathered}
A_{1}=\frac{16 r^{2}+7 r+4}{12 r^{2}+12 r+3} \quad A_{2 \rightarrow 3}=\frac{8 r+1}{(2 r+1)^{2}} \\
B_{1}=0 \quad B_{2 \rightarrow 3}=\frac{3}{2 r+1} \\
A_{4 \rightarrow 5}=r A_{2 \rightarrow 3} \quad B_{4 \rightarrow 5}=r B_{2 \rightarrow 3}
\end{gathered}
$$

with a speed of sound, $c_{s, e}$, defined through $\gamma$ :

$$
\gamma=\frac{c_{s, e}^{2}}{c_{s}^{2}}=\frac{1}{3} \frac{8 r+1}{2 r+1}
$$

and for D2Q13:

$$
\begin{gathered}
A_{1}=\frac{2 \gamma}{3}\left[\frac{4+8 r-3 r^{2}}{2+3 r+r^{2}}\right] \quad A_{2 \rightarrow 5}=\frac{12}{6+7 r-r^{3}} \\
B_{1}=0 \quad B_{2 \rightarrow 5}=\frac{6-4 r}{3-r} \quad B_{6 \rightarrow 9}=\gamma \\
A_{6 \rightarrow 9}=r A_{2 \rightarrow 5} \quad A_{10 \rightarrow 13}=r A_{6 \rightarrow 9} \quad B_{10 \rightarrow 13}=r B_{6 \rightarrow 9}
\end{gathered}
$$

with a speed of sound defined through the ratio $\gamma$ :

$$
\gamma=\frac{c_{s, e}^{2}}{c_{s}^{2}}=\frac{2}{3-r}
$$

In Table 2 tabulated values of the coefficients $A_{i}$ and $B_{i}$ are found as function of the values of $\gamma$ and $r$ used in Section $3 \& 4$

As can be seen, all coefficients $A_{i}=B_{i}=\gamma=1$ for $r=1$ which corresponds to the conventional case as is expected. 


\begin{tabular}{|c|c|c|c|c|c|c|}
\hline$\gamma$ & $r$ & $A_{1}$ & $A_{2 \rightarrow 3}$ & $A_{4 \rightarrow 5}$ & $B_{2 \rightarrow 3}$ & $B_{4 \rightarrow 5}$ \\
\hline \hline 0.8 & 0.4375 & 0.96 & 1.28 & 0.56 & 1.6 & 0.7 \\
\hline 1 & 1 & 1 & 1 & 1 & 1 & 1 \\
\hline 1.2 & 3.25 & 1.16 & 0.48 & 1.56 & 0.4 & 1.3 \\
\hline
\end{tabular}

(a) D1Q5

\begin{tabular}{|c|c|c|c|c|c|c|c|c|}
\hline$\gamma$ & $r$ & $A_{1}$ & $A_{2 \rightarrow 5}$ & $A_{6 \rightarrow 9}$ & $A_{10 \rightarrow 13}$ & $B_{2 \rightarrow 5}$ & $B_{6 \rightarrow 9}$ & $B_{10 \rightarrow 13}$ \\
\hline \hline 0.8 & 0.5 & 1.07 & 1.28 & 0.64 & 0.32 & 1.6 & 0.8 & 0.4 \\
\hline 1 & 1 & 1 & 1 & 1 & 1 & 1 & 1 & 1 \\
\hline 1.2 & 1.33 & 0.84 & 0.92 & 1.23 & 1.65 & 0.4 & 1.2 & 1.6 \\
\hline
\end{tabular}

(b) D2Q13

Table 2: Tabulated values of coefficients $A_{i}$ and $B_{i}$ for use with a modified equilibrium distribution.

\subsection{Numerical stability}

Although we did not carry out a formal stability analysis, we do note that for the equilibrium distribution to retain a positive value, it is required that $r$ must be a positive value, i.e. $r>0$. This is clearly shown in Fig. 2 where the magnitude of the equilibrium distribution is evaluated as function of $r$ for the D1Q5 lattice. A similar analysis can be performed for D2Q13. It follows that the theoretical limits of $\gamma$ for the D1Q5 lattice is $\frac{1}{3}<\gamma<\frac{4}{3}$ while for the D2Q13 lattice is $0<\gamma<\frac{2}{3}$. The implication is that for the D1Q5 lattice only a factor two difference in speeds of sound may be achieved. For the D2Q13 lattice, this is less strict as the speed of sound can be brought to almost zero. However, numerical stability may become an issue as changing $\gamma$ implies varying the Mach number which should be kept small to be consistent with the low Mach number expansion of the equilibrium distribution in Eq. 13.

We have studied the range of stability of the method by conducting simulations with very small Mach numbers, i.e. $\mathrm{Ma}=\frac{u_{m a g}}{c_{s, e}} \ll 1$, in both one and two dimensions. The test cases are described in the next section. By systematically varying the sound speed in these test cases, we found that simulations are stable in the ranges of approximately $0.7<\gamma<1.25$ for D1Q5 and $0.5<\gamma<1.2$ for D2Q13. Since the test cases are all restricted to small Ma, these stability limits do not have any relation whatsoever with the low Mach number approximation which is about ignoring the $O\left(u^{3}\right)$ and higher terms in the equilibrium moments (see Eq. 15) of the equilibrium distribution Eq. 13

We are aware that the D1Q5 and D2Q13 lattices used in this work are known 

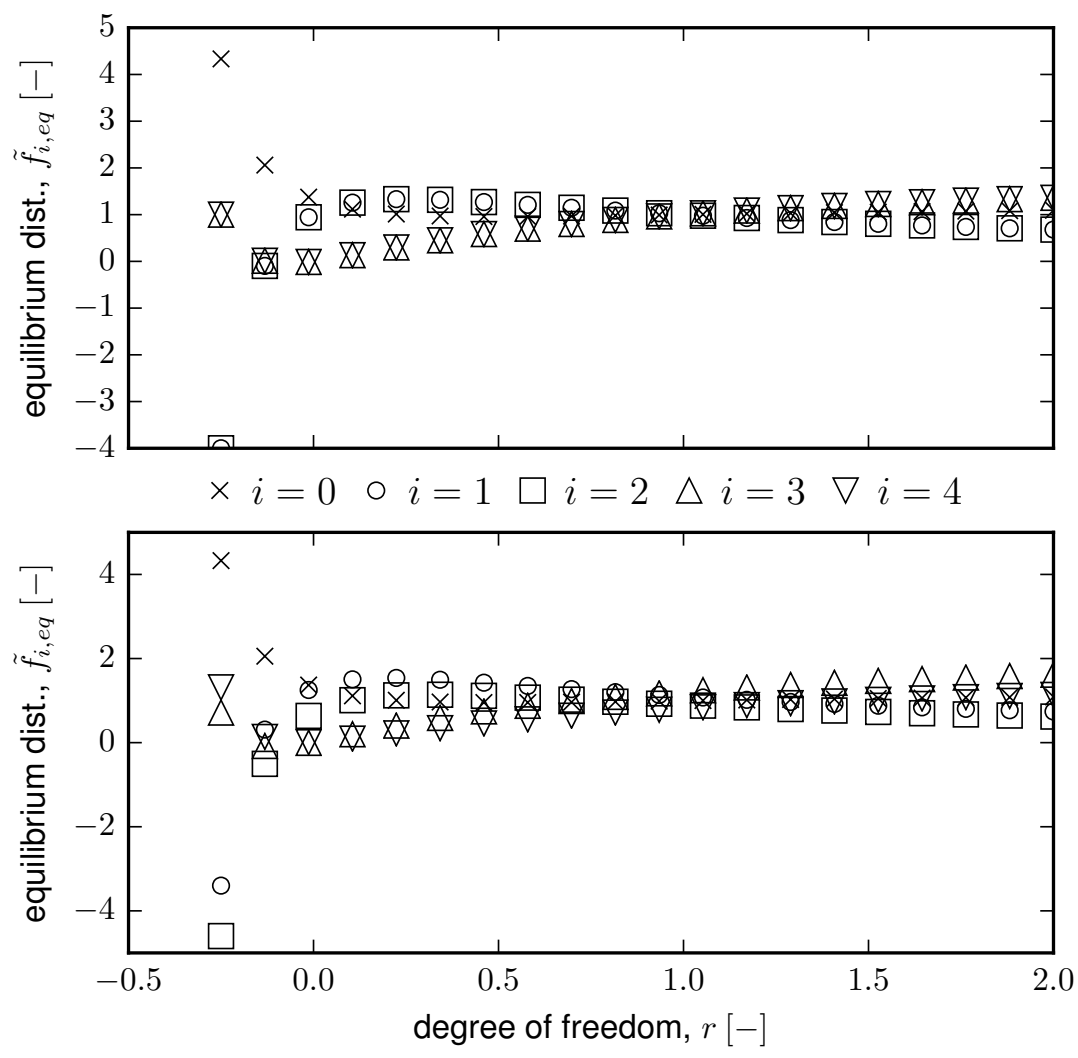

Figure 2: A stability analysis of the modified dimensionless equilibrium distribution $\tilde{f}_{i, e q}=$ $\frac{f_{i, e q}}{w_{i} \rho}$ as a function of degree of freedom $r$ for D1Q5. The top figure shows the values of the equilibrium function for no flow, while the bottom figure shows the values for a net flow of $v=0.1\left[\frac{l u}{l t}\right]$ which is near the stability limit. For the method to remain stable requires the equilibrium distribution to remain positive. 
to be unstable outside of a narrow viscosity range due to entropy considerations [26-28]. However, it was found that these lattices are completely Galilean invariant and more accurate than conventional lattices [28, 29]. Despite these instability concerns, the D2Q13 lattice has recently been used in work related to incompressible flow of the Rayleigh-Taylor instability using a modified equilibrium distribution [30]. Considering the above, we view these lattices suitable for the work presented here if we relax the entropy requirement and limit our work to within this viscosity range.

\subsection{Avoiding loss of precision for low Ma flows}

It was shown by Skordos [31], that for low Mach number flows, significant loss of precision can be observed. This is due to the limited representation of floatingpoint numbers for certain arithmetic operations in the LBM implementation, specifically on the equilibrium distribution. If the density varies around a mean value $\rho_{0}$ with an amplitude $\rho_{0} \Delta \sim M a^{2}$ according to:

$$
\rho=\rho_{0}(1+\Delta)
$$

where $\Delta$ is the grid cell size, then the higher order $M a$ terms in the equilibrium distribution:

$$
f_{i, e q}=w_{i} \rho[1+O(M a)] \approx w_{i} \rho_{0}[1+O(M a)]
$$

become insignificant leading to loss of precision on the density.

To improve the precision, Skordos [31] proposed to decompose the distribution function into a 'rest' equilibrium contribution $f_{i, e q}^{0}=f_{i, e q}\left(\rho_{0}, 0\right)=w_{i} \rho_{0}$ and a perturbative contribution $\delta f_{i}$ :

$$
f_{i}=f_{i, e q}^{0}+\delta f_{i}
$$

The new LBM equation then becomes:

$$
\frac{\delta f_{i}^{\prime}-\delta f_{i}}{\Delta t}=-\frac{\delta f_{i}-\delta f_{i, e q}}{\tau}
$$

which governs the evolution of the perturbation functions rather than the full distribution functions. Taking moments of the decomposed distributions one finds that:

$$
\sum_{i} \delta f_{i}=\delta \rho \quad \sum_{i} \vec{e}_{i} \delta f_{i}=\rho \vec{u}
$$


The perturbation equilibrium distribution is now given by:

$$
\delta f_{i, e q}=f_{i, e q}-f_{i, e q}^{0}=w_{i}\left[\rho_{0} \Delta+\rho O(M a)\right]
$$

which only comprises higher order terms in $M a$ thus leading to an increased numerical precision. This decomposition is implemented for the Taylor-Green vortex discussed further on the paper where Ma decreases exponentially as the number of lattice nodes is increased.

\section{Validation for a standing wave}

The simplest benchmark case for the validation of the implementation of the variable speed of sound is that of a $1 \mathrm{D}$ isothermal standing pressure wave which is very gradually damped due to a very low viscosity [32]. The simulation is done for a periodic domain with a sinusoidal density function as initial condition:

$$
\rho(x, 0)=\rho_{0}+\triangle \rho \sin (k x)
$$

with $\rho_{0}$ the average density, $\triangle \rho$ the amplitude of the wave and $k=\frac{2 \pi}{n}$ the wave number based on the wave length $n$.

We assume that from the very beginning the amplitude of the wave is small enough to satisfy the condition that $\triangle \rho / \rho_{0} \ll 1$. This allows for the linearization of continuity equation and Navier-Stokes equations. In addition, the bulk viscosity may be ignored under this restriction. Upon defining a perturbed density $\rho=\rho_{0}+\rho^{\prime}$ and velocity $u=u^{\prime}$, the solution to the resulting wave equation is the real part (denoted by $\Re$ ) of the following density and velocity functions:

$$
\begin{aligned}
& \rho(x, t)=\rho_{0}+\Delta \rho \sin (k x) \Re\left[\exp \left(-\omega k c_{s, e} t\right)\right] \\
& u(x, t)=-c_{s, e} \frac{\Delta \rho}{\rho_{0}} \cos (k x) \Re\left[\omega \exp \left(-\omega k c_{s, e} t\right)\right]
\end{aligned}
$$

where $\omega=\omega_{r}+i \omega_{i}$ is the dimensionless complex angular velocity with $\omega_{r}$ and $\omega_{i}$, its real and imaginary part, respectively given by:

$$
\omega_{r}=\frac{k \nu_{e}}{c_{s, e}} \quad \omega_{i}= \pm \sqrt{1-\left(\frac{k \nu_{e}}{c_{s, e}}\right)^{2}}
$$

The parameter which characterizes the amount of decay is the real part of the angular velocity, $\omega_{r}$, which may be regarded as a Knudsen number [23] or as 
a type of reciprocal Reynolds number and in either way is the non-dimensional quantity that characterizes the flow. For the above derivations, it was assumed that $\omega_{r} \ll 1$, such that viscous effects remain small. This assumption on $\omega_{r}$ is also required for $\omega_{i}$ to remain real.

A standing wave is simulated in a periodic domain of $n=256$ lattice nodes with $\omega_{r}=10^{-2}$, reference density $\rho_{0}=1$ and the amplitude $\triangle \rho=10^{-3}$ for different values of $\gamma$. Initialization was done with the help of the equilibrium distribution of Eq. 13 and by using the density and velocity fields of Eq. 37 at time $t=0$. Although initializing with the equilibrium distribution might introduce initialization errors for initially transient flows via the viscous stress tensor of Eq. 17 [23, 33], our assumption of a low density amplitude, $\frac{\Delta \rho}{\rho_{0}} \ll 1$, leads to negligibly small gradients in the velocity field and therefore to negligibly small initialization errors. Since $\omega_{r}$ is fixed and $c_{s, e}$ changes as function of $\gamma$, the viscosity $\nu_{e}$ must also change to simulate the same system for D1Q3 and D1Q5. This is done by varying the relaxation time $\tau$ according to Eq. 12

$$
\tau=\frac{1}{2}+\frac{\nu_{e}}{c_{s, e}^{2}}=\frac{1}{2}+\frac{\omega_{r}}{k c_{s, e}}
$$

The wave is allowed to oscillate until the dimensionless time $k c_{s, e} t=50$; for this period, the time-dependent amplitude of the density and velocity as a function of the time is compared to the analytical solutions. For a properly scaled system at a fixed value of $\omega_{r}$ and over the same dimensionless time period, the solutions for both D1Q3 and D1Q5 and the analytical solutions should collapse onto the same curves. In Figure 3 , it is shown that, for $\gamma=1$, the numerical solution for both the D1Q3 and D1Q5 lattice exactly coincides with the analytical solution. Figures 4 and 5 show that for $\gamma \neq 1$, the D1Q3 lattice starts deviating from the analytical curve towards the end of the simulation whereas the D1Q5 lattice is seen to keep following the analytical solution even for long times. Although one could argue that the simulations for $\gamma \neq 1$ in the D1Q3 are redundant because they are known to be deficient, they do show the impact of this deficiency and help illustrating the positive effect of switching to D1Q5 with the view of dealing with $\gamma \neq 1$.

The figures show that, as time progresses, the D1Q3 scheme results in increasing deviations from the analytically calculated amplitude whenever $\gamma \neq 1$ 

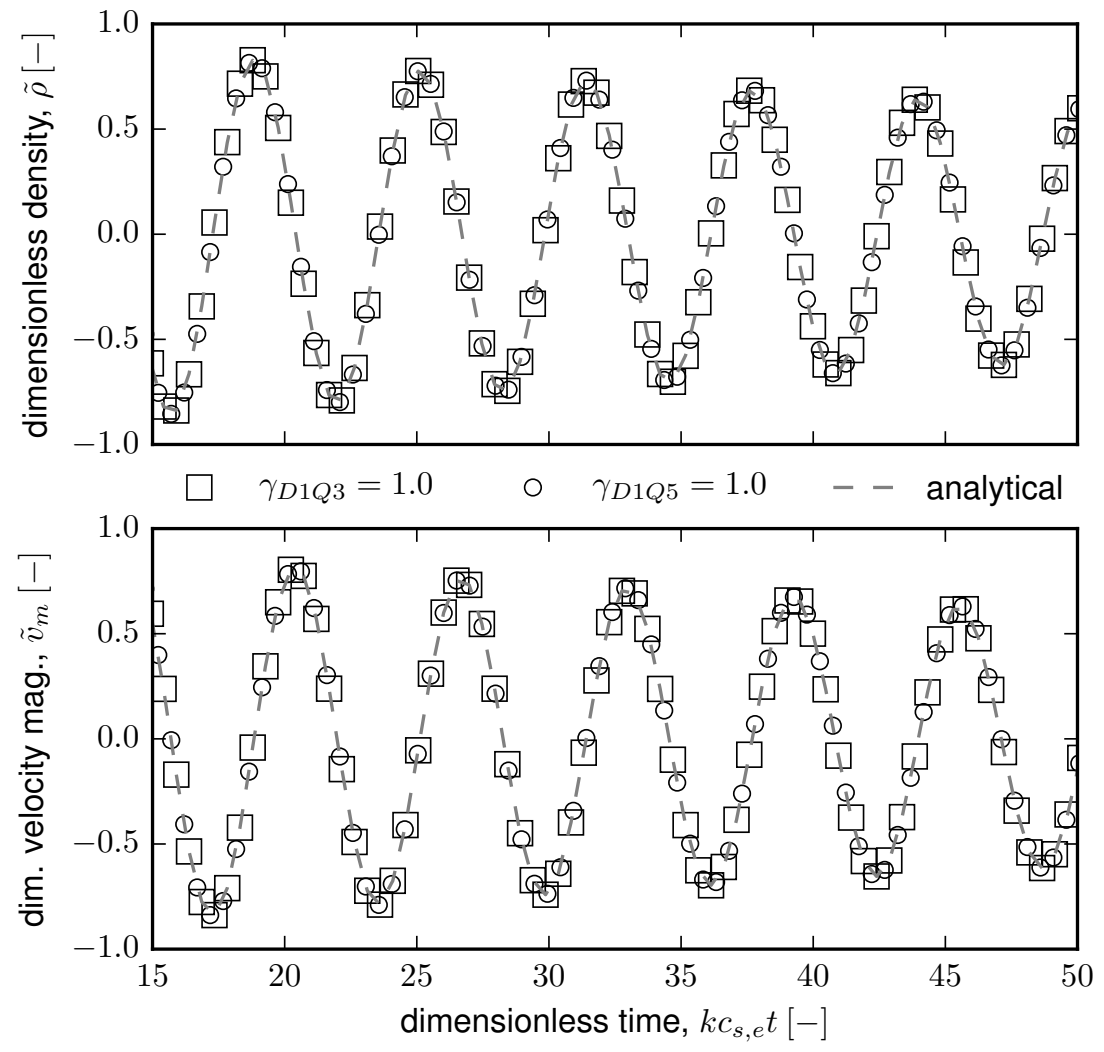

Figure 3: The dimensionless amplitude of the density (top) and velocity magnitude (bottom) for the standing wave at $x=\frac{n}{4}$ with gridsize $n=256$ as a function of non-dimensional time (up to $k c_{s, e} t=50$ ). The amplitudes for $\gamma=1$ for the D1Q3 and D1Q5 lattices are compared to the analytical solution and are shown to coincide exactly as is to be expected for $\gamma=1$. 

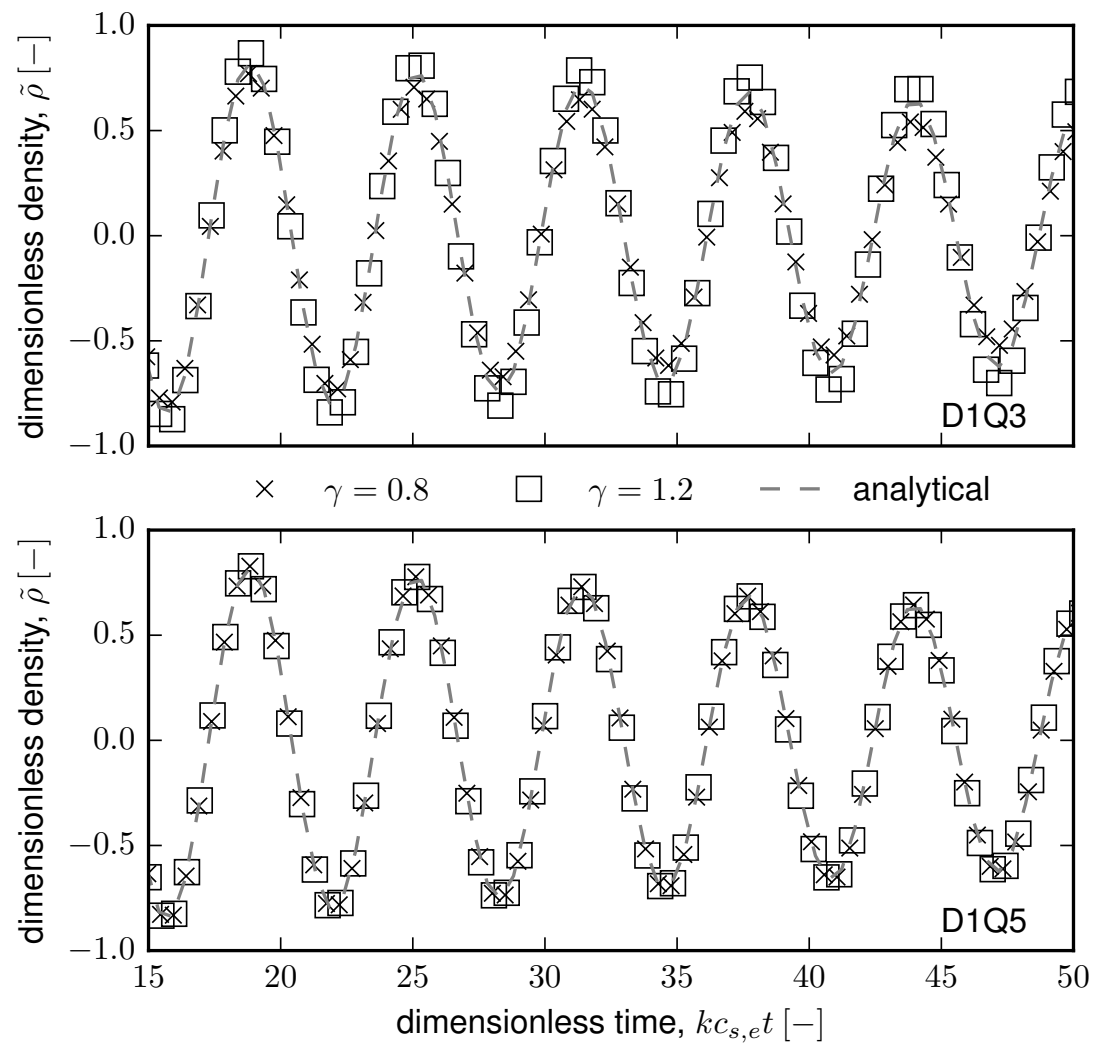

Figure 4: The dimensionless amplitude of the density $\left(\tilde{\rho}=\frac{\rho-\rho_{0}}{\Delta \rho}\right)$ for the standing wave at $x=\frac{n}{4}$ with gridsize $n=256$ as a function of non-dimensional time (up to $k c_{s, e} t=50$ ) for the D1Q3 (top) and D1Q5 (bottom) lattices, respectively, for different values of $\gamma$. As time progresses, the implementation for the D1Q3 lattice (top) starts deviating from the analytical curve, whereas for the D1Q5 lattice (bottom) it does not. 

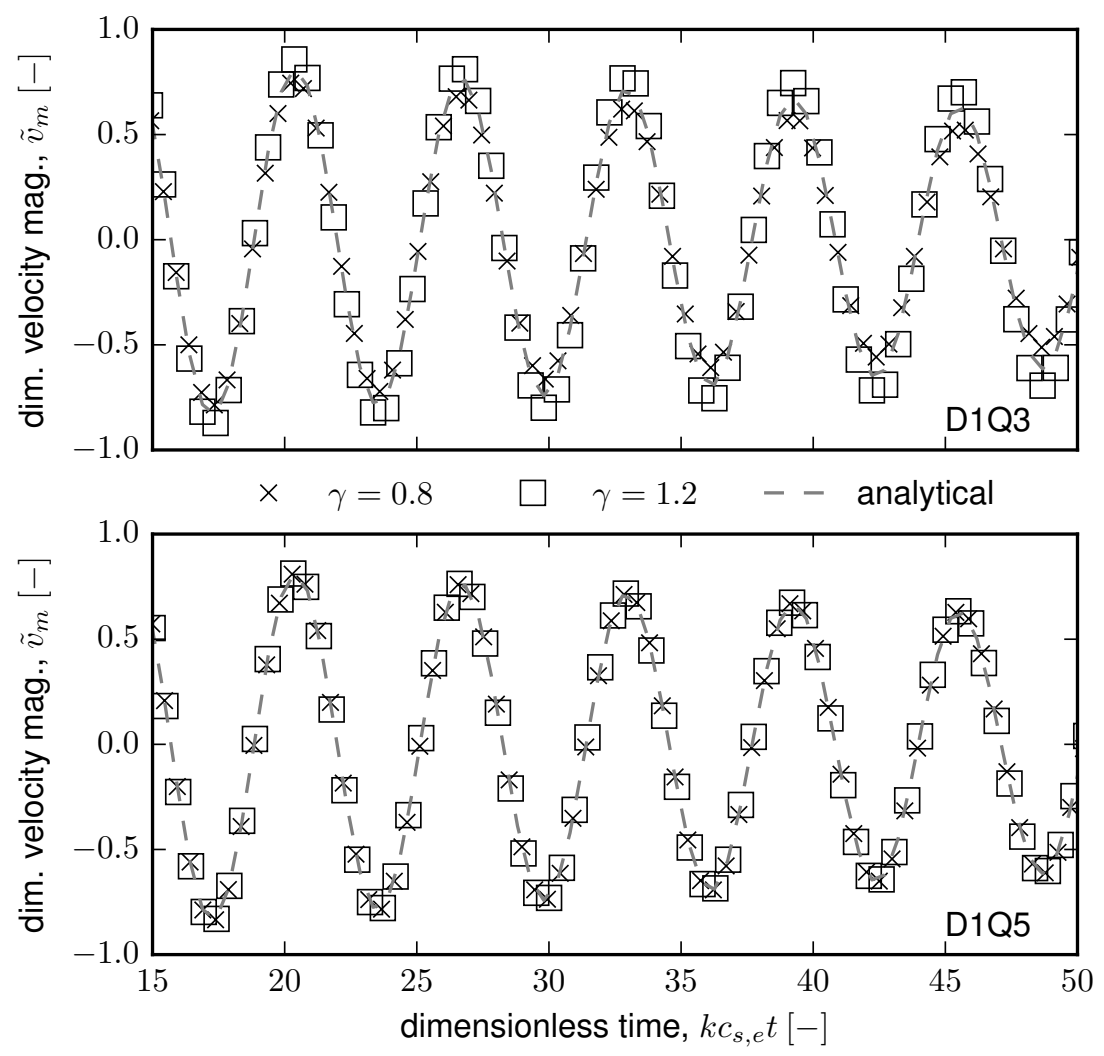

Figure 5: The dimensionless amplitude of the velocity $\left(\tilde{u}=\frac{u}{c_{s, e}} \frac{\rho_{0}}{\Delta \rho}\right)$ for the standing wave at $x=\frac{n}{2}$ as a function of non-dimensional time (up to $k c_{s, e} t=50$ ) for the D1Q3 (top) and D1Q5 (bottom) schemes, respectively, for different values of $\gamma$. Just like in Figure 4 for the amplitude of the density, the implementation for the D1Q3 lattice (top) starts deviating from the analytical curve as time progresses, whereas for the D1Q5 lattice (bottom) it does not. 
whereas the D1Q5 scheme more accurately predicts the peak density for the same range of $\gamma$ values. This becomes more apparent when at the end of each simulation (at dimensionless time $k c_{s, e} t=50$ ) we consider the relative global error of the computed density and velocity fields as defined by the L2-norm [34, 35]:

$$
\epsilon=\sqrt{\frac{\sum_{i}\left(\phi_{i}-\phi_{i, r e f}\right)^{2}}{\sum_{i} \phi_{i, r e f}^{2}}}
$$

where $\phi_{i}$ is either the computed density or the computed velocity magnitude, $\phi_{i, r e f}$ is the reference density or velocity, while the summation is done over all grid nodes.

Fig. 6 shows the relative global errors of the density and velocity fields plotted as a function of $\gamma$. The D1Q5 scheme results in relative global errors which slightly increase with $\gamma$, while for the D1Q3 scheme the relative global errors strongly increase as $\gamma$ is moved away from $\gamma=1$. The D1Q3 behaviour is due to the above discussed inadequacy of the lattice for $\gamma \neq 1$; for the slight increase of the relative global errors in the D1Q5 case we do not have a clear explanation.

A grid convergence analysis in Fig. 7 for the D1Q3 scheme and Fig. 8 for the D1Q5 scheme shows a significant improvement in convergence for the D1Q5 scheme. For D1Q3, the density and velocity global errors remain more or less constant for $\gamma \neq 1$, while for D1Q5 they are observed to have almost second-order convergence irrespective of the value of $\gamma$. It is observed that the relative global error of the velocity field is relatively large for coarse grids. For coarse grids, we found the numerical solution slowly moving out of phase with the analytical solution over time, resulting in relatively large deviations at the point in time at which the two solutions are compared. Clearly, this improves when refining the grid.

Our results in Fig. 7 deviate from data reported by Viggen [3] which show a second-order convergence for the D1Q3 lattice while varying the speed of sound. As a matter of fact, the model proposed by Viggen, though fundamentally similar, is different from the conventional D1Q3 model. The method proposed by Viggen allows the speed of sound to be tuned by forcing constraints on the equilibrium distribution function without the need of an extended velocity set on a 1D lattice. Unfortunately, Viggen's method cannot be implemented for 2D 

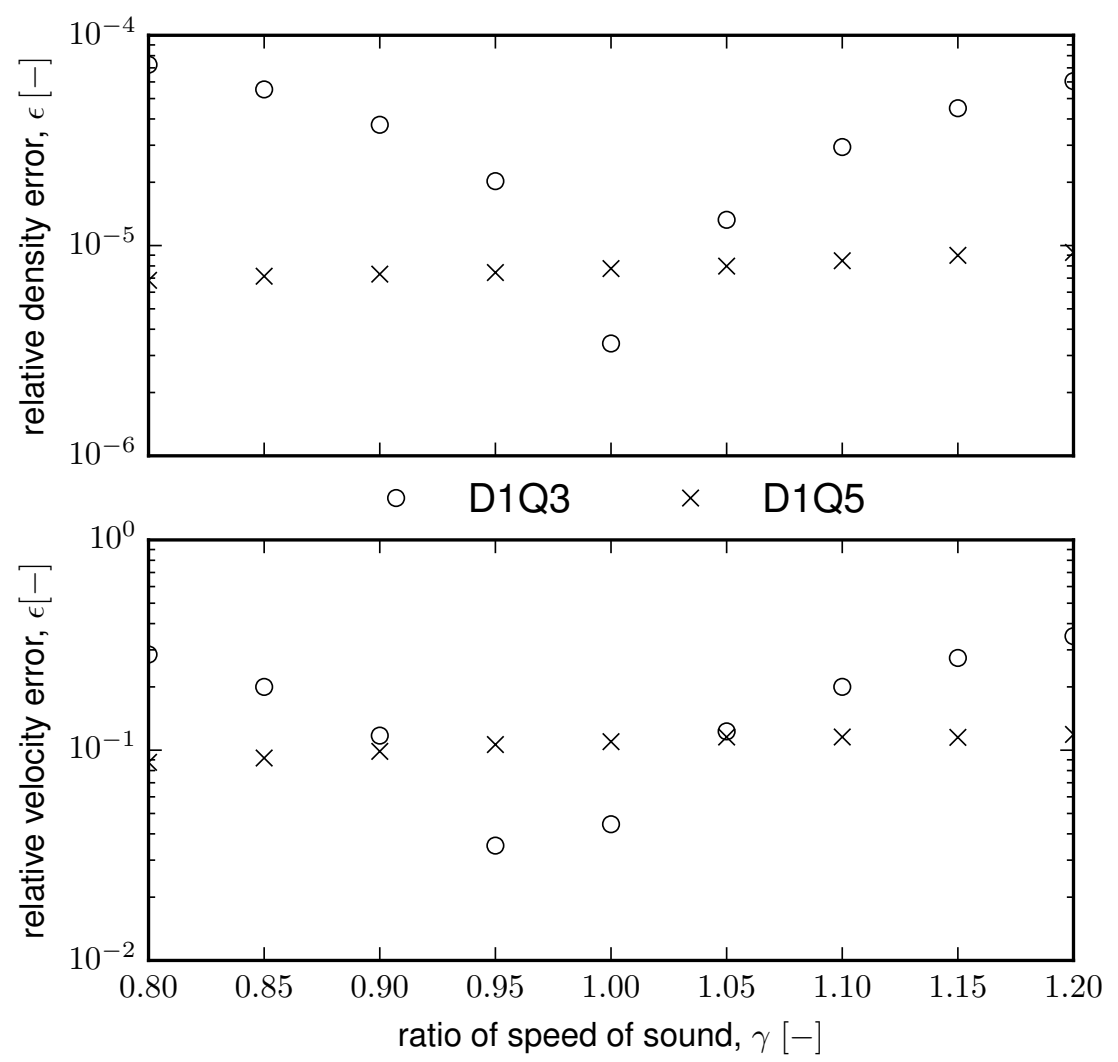

Figure 6: The relative global error in the density and velocity for the D1Q3 vs D1Q5 lattices for a damped standing wave as a function of $\gamma$ for gridsize $n=256$. The errors for the D1Q5 implementation increase only slightly with $\gamma$ but overall are lower than for the D1Q3 implementation except for $\gamma=1$ when the errors are lower for D1Q3 than for D1Q5

and 3D flows. We, however, exploit a modified equilibrium distribution function leading to the necessity of extended velocity sets such that we can vary the speed of sound and easily extend this method to higher dimensions.

\section{Validation for the Taylor-Green vortex}

For a two-dimensional test case, we validated our D2Q13 scheme for the Taylor-Green Vortex (TGV) which has been used before as a benchmark by several authors, e.g. [14, 20, 36, 37]. While in many cases the interest in TGVs is related to the transition to turbulence and the LBM simulations are therefore carried out at higher Reynolds numbers, our focus is on a TGV under viscous 

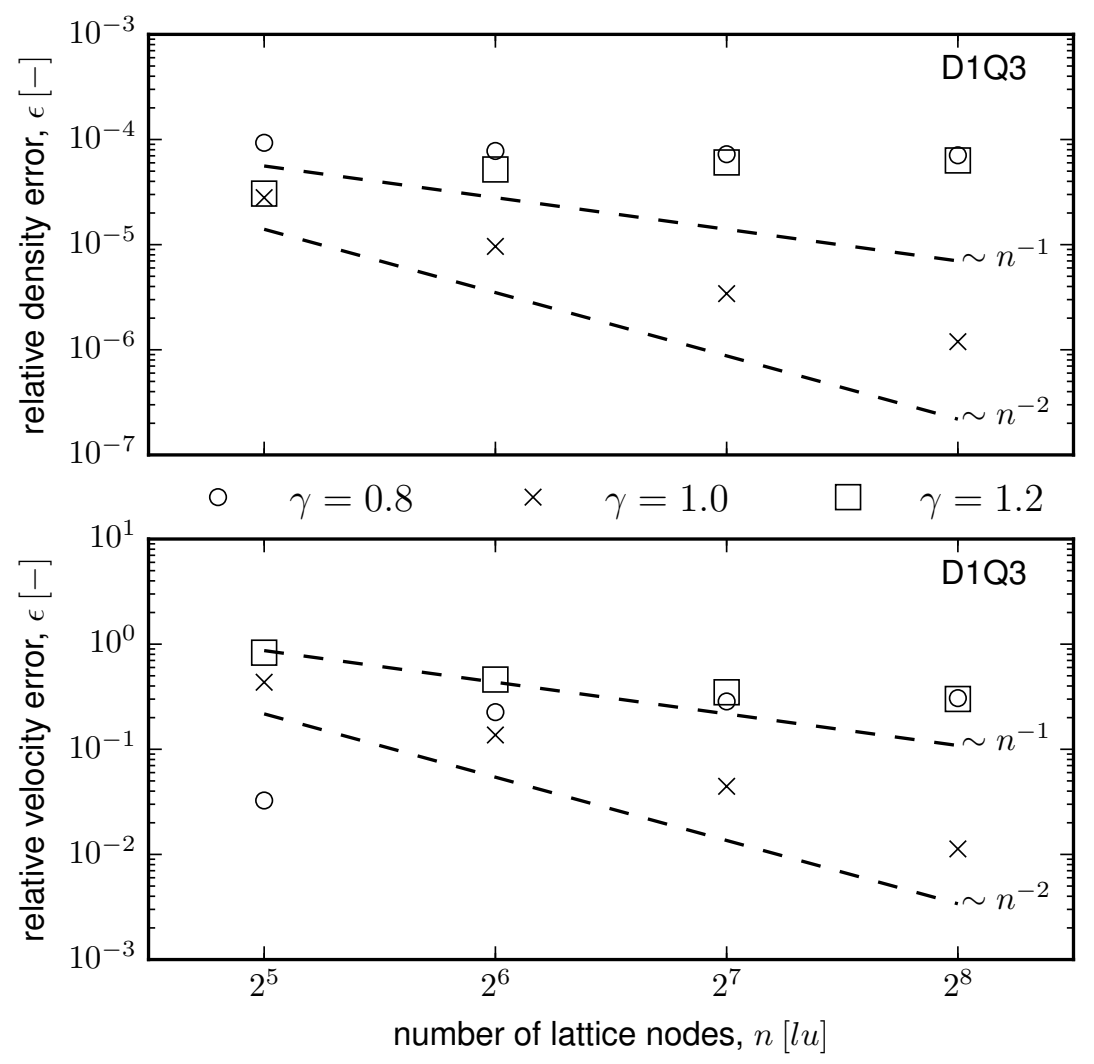

Figure 7: The relative global error in density and velocity fields for D1Q3 for the standing wave as a function of the number of lattice nodes used, for different values of $\gamma$. The dashed lines represent the order of the error. This clearly shows large deviations for $\gamma \neq 1$. 

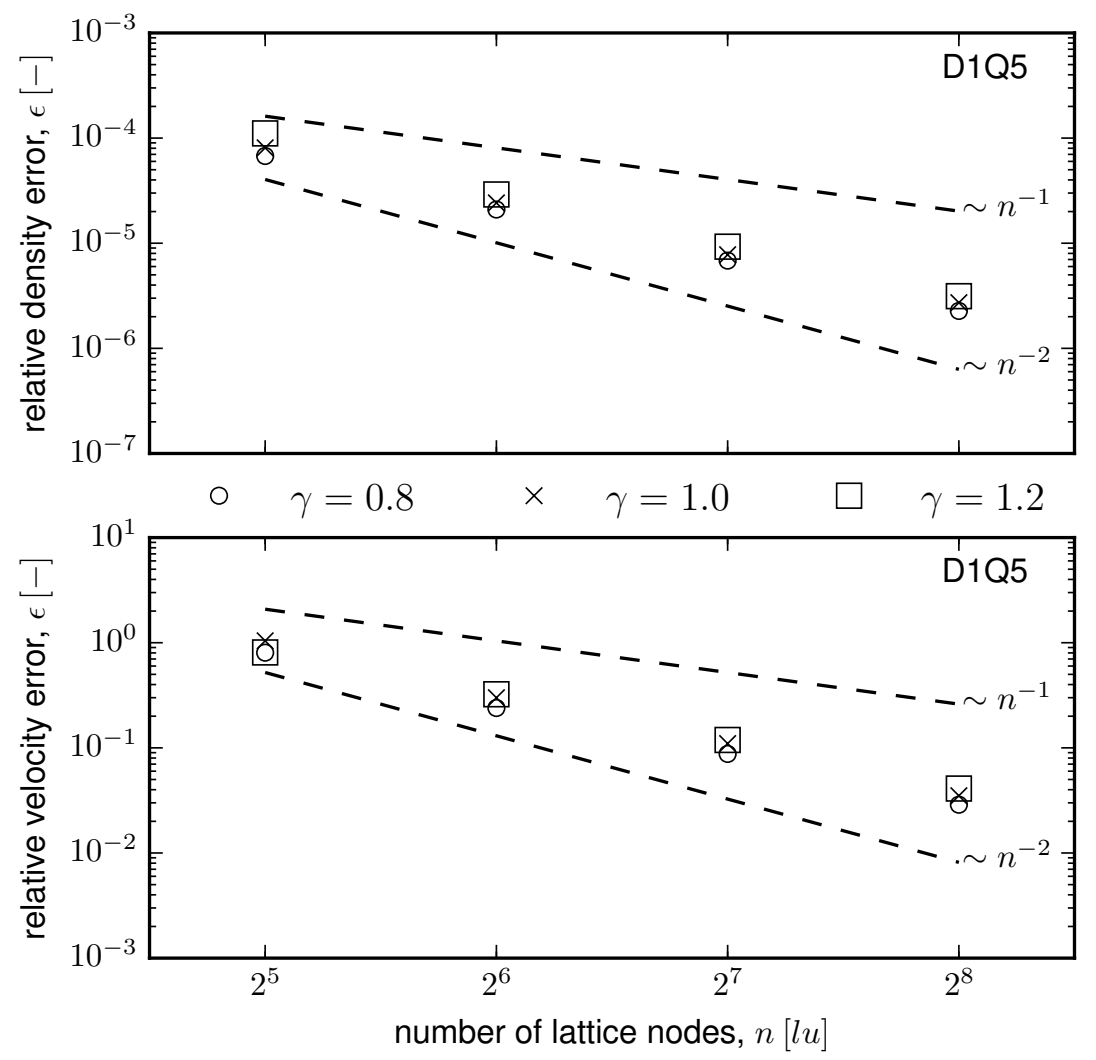

Figure 8: The relative global error in density and velocity fields for D1Q5 for the standing wave as a function of the number of lattice nodes used, for different values of $\gamma$. The dashed lines represent the order of the error. This clearly shows closer agreement than for the D1Q3 implementation in Fig 7 for $\gamma \neq 1$. 
conditions, i.e. at a very low Reynolds number. The initial condition of a TGV in terms of density and velocity fields is illustrated in Fig. 9 that demonstrates the periodic character of the flow. The TGV then decays due to viscosity. For the transient behavior of this TGV, exact analytical expressions for the velocity and pressure fields obeying the classical incompressible Navier-Stokes equation are available:

$$
\begin{gathered}
p(x, y, t)-p_{0}=\frac{1}{8} \rho u_{0}^{2}[\cos (2 k x)+\cos (2 k y)] \exp \left(-4 k^{2} \nu t\right) \\
u(x, y, t)=-\frac{u_{0}}{\sqrt{2}} \cos (k x) \sin (k y) \exp \left(-2 k^{2} \nu t\right) \\
v(x, y, t)=-\frac{u_{0}}{\sqrt{2}} \sin (k x) \cos (k y) \exp \left(-2 k^{2} \nu t\right)
\end{gathered}
$$

where $p_{0}$ and $u_{0}$ are an arbitrary reference pressure and velocity magnitude respectively and the wave number for a square domain of size $n$ is given by $k=2 \pi / n$.

Since Eq. 1 recovers the hydrodynamic equations in the low Mach number limit, i.e. $\mathrm{Ma} \equiv \frac{u_{0}}{c_{s, e}} \ll 1$, the density fluctuates about a mean density $\rho_{0}$ according to $\rho=\rho_{0}+\triangle \rho$ where $\frac{\Delta \rho}{\rho_{0}} \sim \mathrm{Ma}^{2}$. We made sure the Mach number remains small by making sure $u_{0} \ll c_{s, e}$. The LBM equation of state $p=\rho c_{s, e}^{2}$, results in the evolution of the density field is equivalent to that of the pressure field:

$$
\rho(x, y, t)=\rho_{0}\left[1+\frac{1}{8} \mathrm{Ma}_{0}^{2}[\cos (2 k y)+\cos (2 k x)] \exp \left(-4 k^{2} \nu_{e} t\right)\right]
$$

where $\mathrm{Ma}_{0}=\frac{u_{0}}{c_{s, e}}$ is the initial Mach number.

The TGV was simulated in a periodic square domain with the initial conditions:

$$
\begin{gathered}
\rho(x, y, 0)=\rho_{0}\left[1+\frac{1}{8} \mathrm{Ma}^{2}[\cos (2 k y)+\cos (2 k x)]\right] \\
u(x, y, 0)=-\frac{u_{0}}{\sqrt{2}} \cos (k x) \sin (k y) \\
v(x, y, 0)=-\frac{u_{0}}{\sqrt{2}} \sin (k x) \cos (k y)
\end{gathered}
$$

where the LBM parameters were set as $\rho_{0}=1$ and $\tau=1$ for $\operatorname{Re}=10^{-1}$. The square domain is of length $n \Delta x$, where $n$ is the number of grid points per dimension in the range $[128,1024]$, and $\Delta x=1$ is the lattice spacing. 

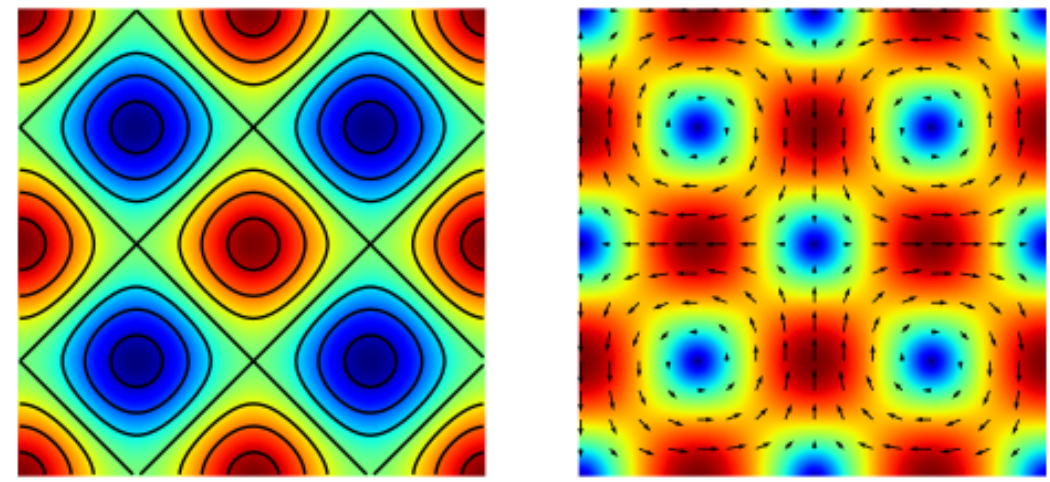

Figure 9: Initial conditions of a Taylor-Green Vortex: density (left), velocity (right).

From the Reynolds number the velocity magnitude of the vortex, $u_{0}$, is calculated. The initialization was done in the same way as in the previous case of the damped standing wave, i.e. with the equilibrium distribution of Eq. 13 on the basis of identical arguments: the initial gradients in the velocity fields are relatively small. We did check whether including the non-equilibrium populations in the initialization made a difference for the transient behaviour of the TGV in our simulations: we found it did not.

The vortex was subsequently allowed to decay for one characteristic time scale, i.e. $k^{2} \nu_{e} t=1$. Initially, simulations were carried out for the number of lattice nodes equal to $n$ of 16,32,64 and 128 and for values of $\gamma$ between 0.8 and 1.2. It was found that for small $n$, the density field exhibited spurious density oscillations $(\sim 10 \%)$ superimposed on the exact solution in Eq. 42 . We checked whether these spurious oscillations were due to ignoring the non-equilibrium distributions in the initialization step; extensive testing showed however that this was not the case. Further tests using the equilibrium distribution reported by Chai and Zhao [20] resulted in the same behaviour. It was found that these spurious density oscillations disappear when using a finer grid as noted by Chai and Zhao. 20.

For both D2Q9 and D2Q13, the time-dependent amplitudes for density and velocity magnitude as a function of the dimensionless time for $n=1024$ are compared mutually and with the analytical solution in Figs. 10 and 11

On the basis of the analytical solution and a proper scaling, it is expected 

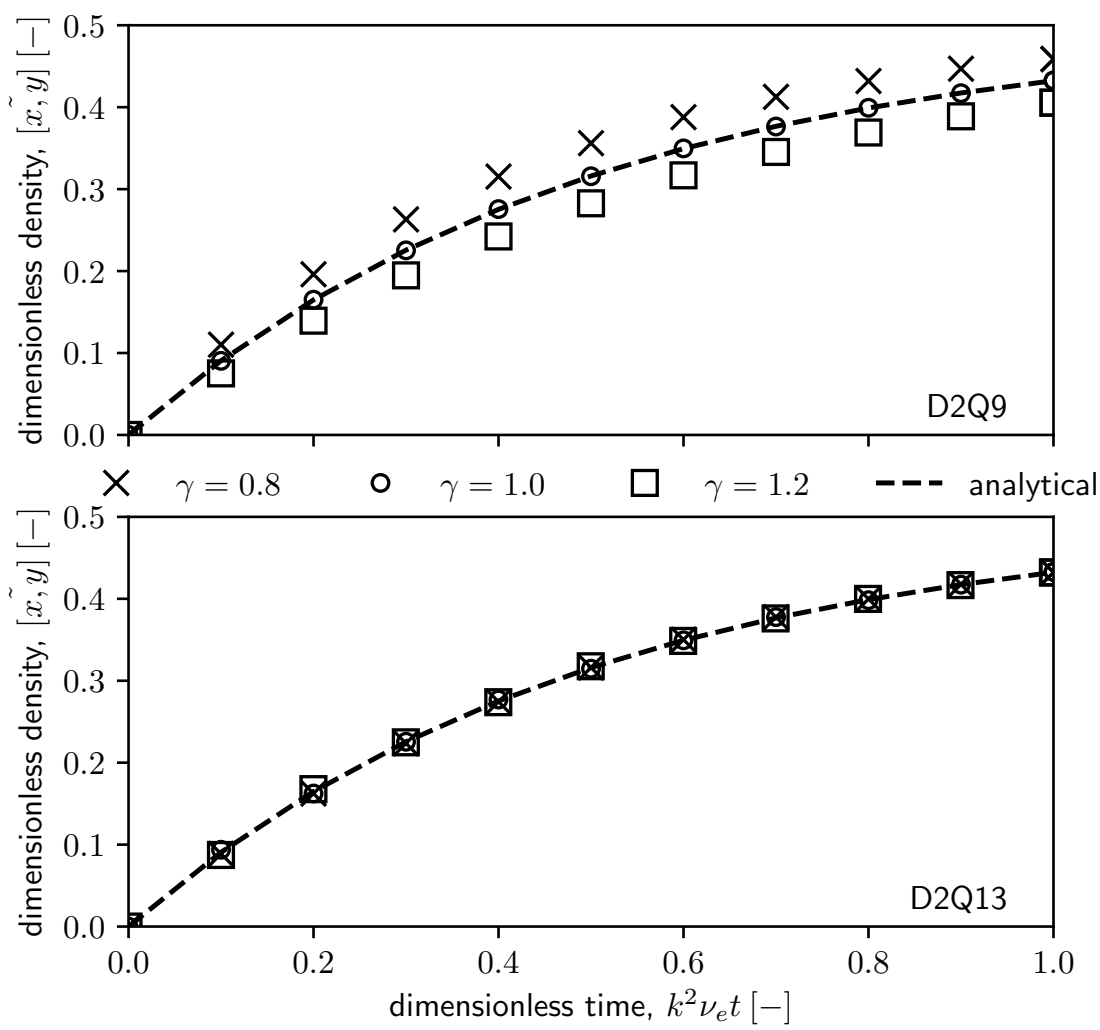

Figure 10: The dimensionless amplitude of the density as a function of the non-dimensional time (up to $k^{2} \nu_{e} t=1$ ) for D2Q9 (top) vs D2Q13 (bottom) schemes, for different values of $\gamma$ at a gridsize $n=1024$. The dashed line is the analytical solution. 

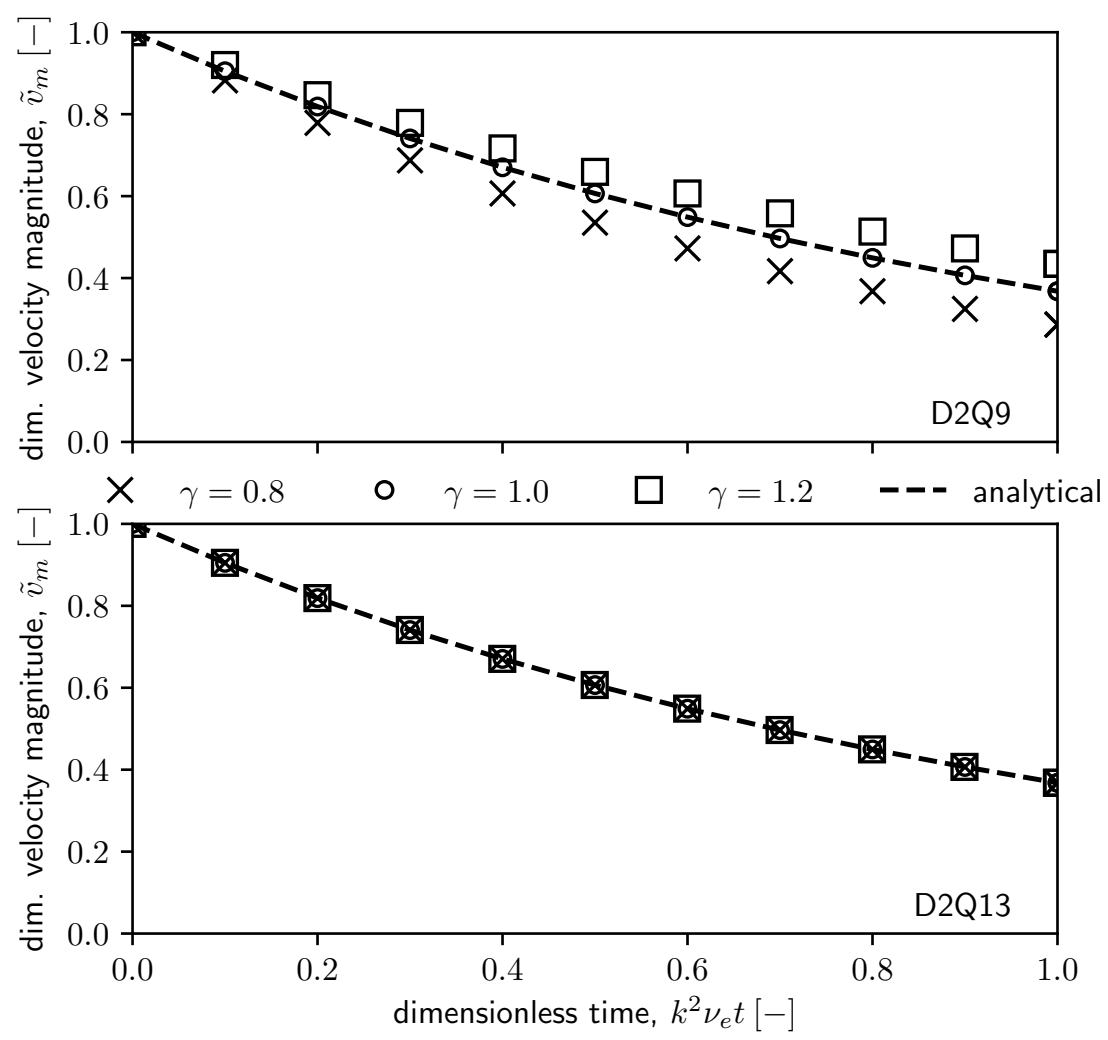

Figure 11: The dimensionless amplitude of the velocity magnitude as a function of the nondimensional time (up to $k^{2} \nu_{e} t=1$ ) for D2Q9 (top) vs D2Q13 (bottom) schemes, for different values of $\gamma$ at a gridsize $n=1024$. The dashed line is the analytical solution. 
that, for all values of $\gamma$ within the stability limits, the decay curves collapse onto a single curve. However, as was the case with the standing wave, the time-dependent dimensionless amplitudes of the density and velocity on the D2Q9 lattice differ significantly from the analytical solution over the range of $\gamma$ values applied and do not collapse onto a single curve (Fig. 10a and Fig. 11a). For the D2Q13 implementation, the curves do collapse (Fig. 10p and Fig. 11p). Although even for $n=1024$ the effect on the relative global error in the density field is limited (Fig. 12a), the relative global error in the velocity field is significantly reduced (again except for $\gamma=1$ ) as shown in Fig. 12p. Specifically, the relative error in the density is found to be several orders of magnitude lower over the simulated range of $\gamma$ values indicating a significant increase in accuracy when varying the speed of sound in a multi-speed D2Q13 lattice versus a single-speed D2Q9 lattice.

A similar analysis with respect to the effect of using different speeds of sound but now as a function of grid size, in the range from $\mathrm{n}=128$ to $\mathrm{n}=$ 1024, resulted in Figures 13 and 14. As seen in Figure 13 , the very low relative density errors for the usual D2Q9 exhibit third-order convergence for $\gamma=1$ only and second-order convergence for $\gamma \neq 1$, while for D2Q13 convergence is third-order irrespective of the value of $\gamma$. In Figure 14 the implementation for D2Q9 shows the relative global error in the velocity field is relatively high and does not decrease by refining the lattice for $\gamma \neq 1$, while for D2Q13 convergence is second-order irrespective of the value of $\gamma$. Also these figures demonstrate the attractiveness of D2Q13 over D2Q9 for $\gamma \neq 1$.

\section{Conclusion}

This paper on varying the speed of sound in LBM simulations is prompted by the observation in the LBM literature that methods for on-lattice propagation with a modified equilibrium distribution on a standard lattice are only adequate for non-flowing systems. As soon as flow is involved, such as in acoustics and with flow chemistry, numerical errors are introduced which severely limit the accuracy of LBM simulations on standard lattices. These errors did not withhold several authors from tuning the sound of speed on standard lattices for cases involving flow. 

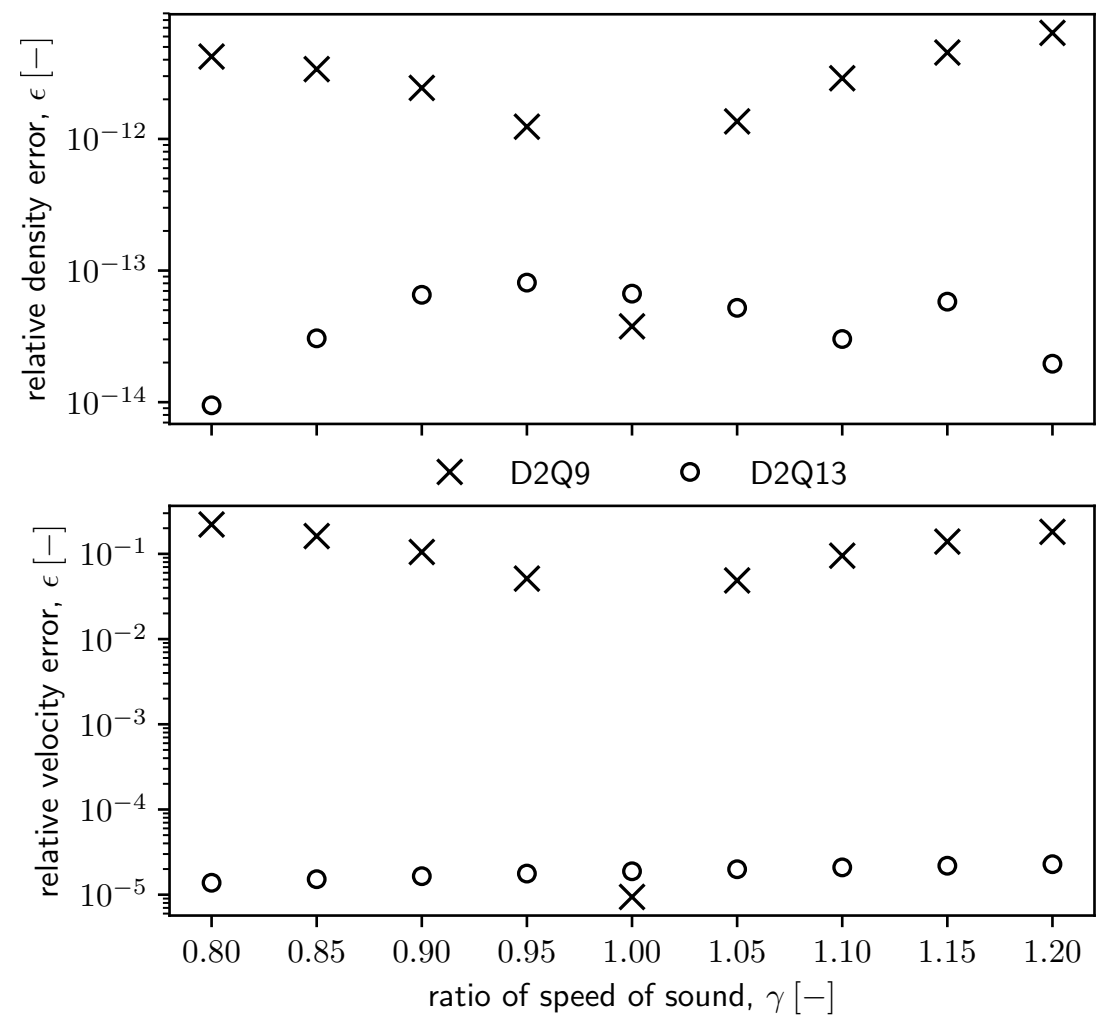

Figure 12: The relative global errors, based on the L2-norm, in the density (top) and velocity (bottom) fields for a Taylor-Green Vortex on the D2Q9 and D2Q13 lattices, respectively, as a function of $\gamma$ for gridsize $n=1024$. 


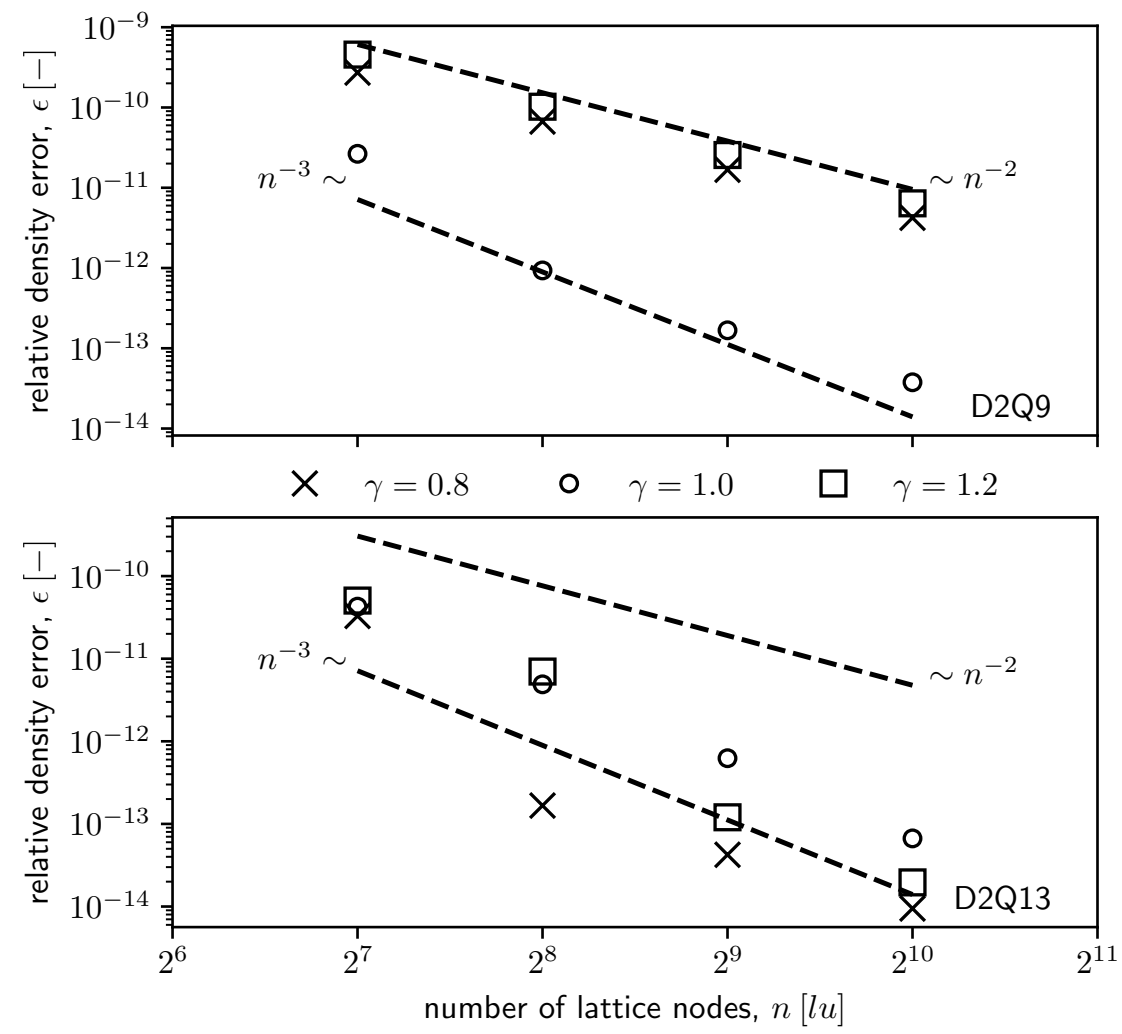

Figure 13: A comparison of the relative global errors in the density fields for a Taylor-Green Vortex, based on the L2-norm, as a function of the number of lattice nodes, for different values of $\gamma$. Top - D2Q9 lattice, Bottom - D2Q13 lattice. The dashed lines represent the order of the error. 

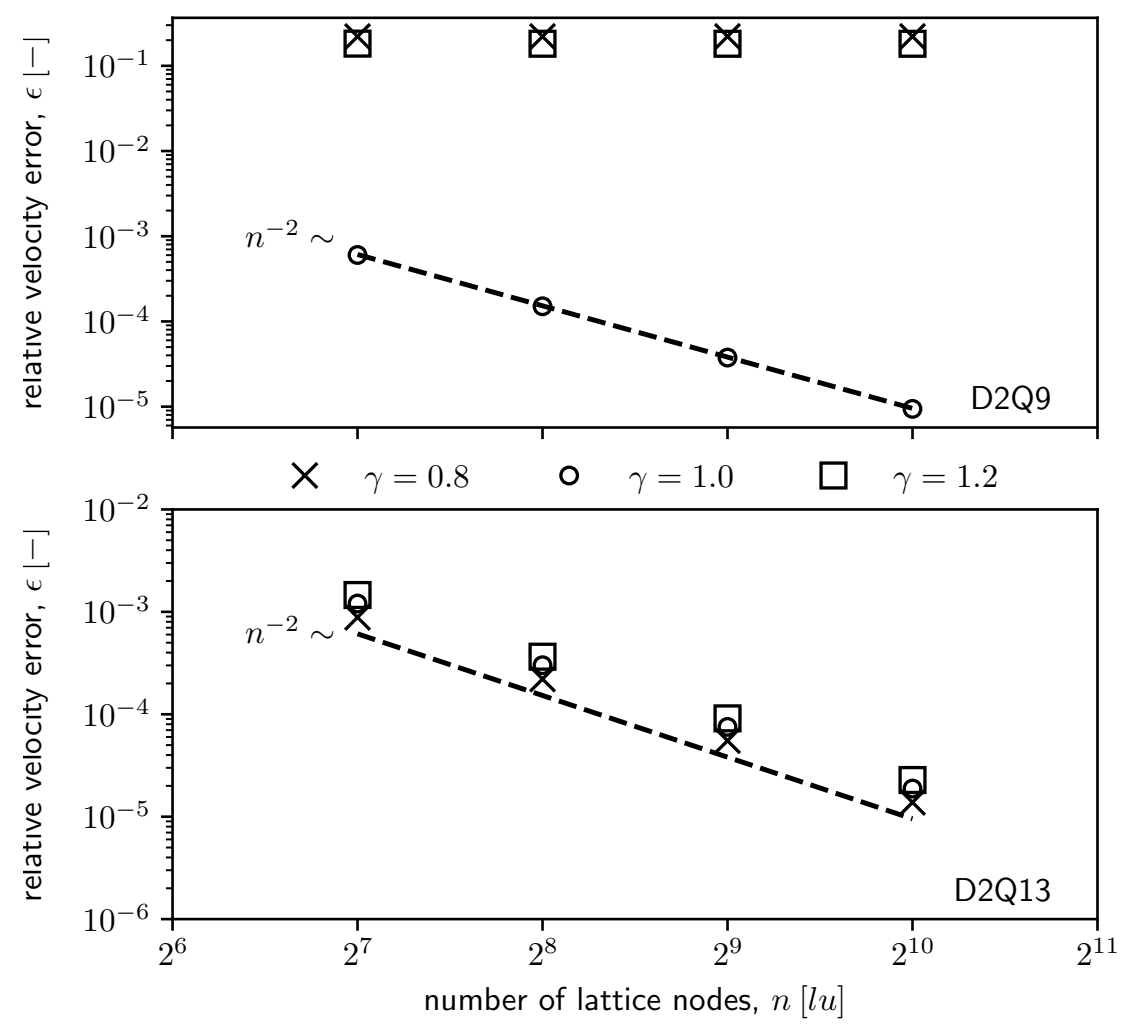

Figure 14: A comparison of the relative global errors in the velocity magnitude fields for a Taylor-Green Vortex, based on the L2-norm, as a function of the number of lattice nodes, for different values of $\gamma$. Top - D2Q9 lattice, Bottom - D2Q13 lattice. The dashed lines represent the order of the error. 
Resolving the issue of tuning the velocity of sound for flows, in which components have a different molecular mass, requires either off-lattice propagation methods or an on-lattice scheme on an extended velocity lattice. Opting for the latter, we extended the velocity set to five, instead of three, for one-dimensional flows and to thirteen, rather than nine, for two dimensional hydrodynamics; these extended velocity sets are referred to as D1Q5 and D2Q13, respectively. This was shown by means of a systematic theoretical treatment, including a derivation to arrive at D1Q5 and D2Q13.

We have shown that the option of allowing for a tunable speed of sound in reactive flows requires modification of the standard equilibrium function along with the use of an extended velocity set and that only then a correct description of the hydrodynamics is retained; this is in contrast to LBM simulations on standard velocity lattices. A simplified stability analysis showed that the stability range for this model yielded a maximum factor of four difference in speed of sound. Subsequently, we carried out a systematic evaluation of the accuracy and range of applicability of this approach and found only marginal errors in the velocity and density fields for two canonical types of isothermal single-phase single-component flow. Thus we have shown that this implementation enables the speed of sound to be varied independently of the grid while the correct hydrodynamics is retained. Our simulations were found to be stable for variations in the speed of sound in the order of two.

The method for variable speeds of sound was verified and validated for simple well-documented benchmark flows, viz. in 1D for a viscously damped standing pressure wave and in 2D for a viscously damped Taylor-Green Vortex. The results for both cases show that for varying speeds of sound, the proposed method outperforms methods on standard velocity lattices: the relative global errors in density and velocity fields are significantly reduced and show second-order convergence for the velocity field on extended velocity lattices. Because of our eventual interest in chemically reactive flows and due to the failure of many LBM schemes to retain the correct hydrodynamics, the focus was on the convergence of the velocity field. At the same time, the convergence of the density field in the proposed method was shown to be comparable to that obtained on a standard lattice and at least second-order accurate. 
The method can be easily incorporated in existing LBM numerical codes by changing the equilibrium distribution. If required, the speed of sound can be varied by a factor of four compared to the original grid speed of sound without introducing large errors in the velocity field compared to the standard lattice. Compared to LBM simulations on a standard lattice, the method is approximately one-third more computationally intensive due to the use of an extended velocity lattice. The performance of the method is estimated to be at least equivalent to that of off-lattice propagation schemes, however with the added benefits of limited code modification (to accommodate the use of a modified equilibrium function) and of conservation of the local nature of the LBM framework allowing for easy parallelization.

In future research, this implementation allows for certain classes of problems such as transport of species with varying molecular masses, in particular in reacting flows which will be the topic of a future paper.

\section{Acknowledgments}

The authors would like to thank Dr. Orest Shardt for his suggestions for improving this work. The research leading to these results has been carried out in the context of a cooperation with the Netherlands Institute for Sustainable Process Technology (ISPT), with support from Shell Amsterdam, as part of ISPT's LBM project PI-20-10. Furthermore, generous sponsoring by Shell Global Solutions at the start of the project is highly appreciated.

\section{Bibliography}

[1] M. R. Kamali, S. Sundaresan, H. E. A. Van den Akker, J. J. J. Gillissen, A multi-component two-phase lattice boltzmann method applied to a 1d fischer-tropsch reactor, Chemical Engineering Journal 207 (SI) (2012) $587-595$.

[2] J. J. J. Gillissen, N. Looije, Boundary conditions for surface reactions in lattice boltzmann simulations, Phys. Rev. E 89 (2014) 063307. URL http://link .aps.org/doi/10.1103/PhysRevE.89.063307 
[3] E. M. Viggen, Acoustic equations of state for simple lattice boltzmann velocity sets, Phys. Rev. E 90 (2014) 013310.

URL http://link.aps.org/doi/10.1103/PhysRevE.90.013310

[4] S. Succi, The Lattice Boltzmann equation: for fluid dynamics and beyond, Oxford University press, 2001.

[5] D. Wolf-Gladrow, Lattice-Gas Cellular Automata and Lattice Boltzmann Models: An Introduction, Springer, New York, 2000.

[6] B. Dünweg, A. J. C. Ladd, Lattice Boltzmann Simulations of Soft Matter Systems, 2009, p. 89.

[7] Y. Qian, Simulating thermohydrodynamics with lattice bgk models Journal of Scientific Computing 8 (3) (1993) 231-242.

URL https://doi.org/10.1007/BF01060932

[8] C. Teixeira, H. Chen, D. M. Freed, Multi-speed thermal lattice boltzmann method stabilization via equilibrium under-relaxation Comp. Phys. Comm. 129 (1-3) (2000) $207-226$.

URL http://www.sciencedirect.com/science/article/pii/ S0010465500001089

[9] Y. Peng, C. Shu, Y. T. Chew, Simplified thermal lattice boltzmann model for incompressible thermal flows, Phys. Rev. E 68 (2) (2003) 026701.

[10] N. Frapolli, S. Chikatamarla, I. V. Karlin, Multispeed entropic lattice boltzmann model for thermal flows, Phys. Rev. E 90 (4) (2014) 043306.

[11] J. Buick, J. Cosgrove, Investigation of a lattice boltzmann model with a variable speed of sound, Journal of Physics A: Mathematical and General 39 (44) (2006) 13807.

URL http://stacks.iop.org/0305-4470/39/i=44/a=013

[12] M. McCracken, J. Abraham, Lattice boltzmann methods for binary mixtures with different molecular weights, Phys. Rev. E 71 (2005) 046704.

URL http://link.aps.org/doi/10.1103/PhysRevE.71.046704 
[13] S. Arcidiacono, J. Mantzaras, I. Karlin, Lattice boltzmann simulation of catalytic reactions, Phys. Rev. E 78 (2008) 046711.

URL http://link.aps.org/doi/10.1103/PhysRevE.78.046711

[14] A. Bardow, I. V. Karlin, A. A. Gusev, Multispeed models in off-lattice boltzmann simulations, Phys. Rev. E 77 (2008) 025701.

URL http://link.aps.org/doi/10.1103/PhysRevE.77.025701

[15] F. Alexander, H. Chen, S. Chen, G. Doolen, Lattice boltzmann model for compressible fluids, Phys. Rev. A 46 (1992) 1967-1970.

URL http://link.aps.org/doi/10.1103/PhysRevA.46.1967

[16] X. Shan, H. Chen, Lattice boltzmann model for simulating flows with multiple phases and components, Phys. Rev. E 47 (1993) 1815-1819.

URL http://link.aps.org/doi/10.1103/PhysRevE.47.1815

[17] X. Shan, G. Doolen, Diffusion in a multicomponent lattice boltzmann equation model, Phys. Rev. E 54 (1996) 3614-3620.

URL http://link.aps.org/doi/10.1103/PhysRevE.54.3614

[18] Y. Guangwu, C. Yaosong, H. Shouxin, Simple lattice boltzmann model for simulating flows with shock wave, Phys. Rev. E 59 (1999) 454-459.

URL http://link . aps .org/doi/10.1103/PhysRevE.59.454

[19] K. Li, C. Zhong, A lattice boltzmann model for simulation of compressible flows, Int. J. Num. Methods in Fluids 77 (6) (2015) 334-357.

URL http://dx.doi.org/10.1002/fld.3984

[20] Z.-H. Chai, T.-S. Zhao, A pseudopotential-based multiple-relaxation-time lattice boltzmann model for multicomponent/multiphase flows, Acta Mechanica Sinica 28 (4) (2012) 983-992.

URL http://dx.doi .org/10.1007/s10409-012-0123-6

[21] P. L. Bhatnagar, E. P. Gross, M. Krook, A Model for Collision Processes in Gases. I. Small Amplitude Processes in Charged and Neutral OneComponent Systems, Physical Review 94 (1954) 511-525.

[22] J. Latt, Hydrodynamic limit of lattice boltzmann equations, Ph.D. thesis (03/09 2007). 
[23] E. M. Viggen, The lattice boltzmann method: Fundamentals and acoustics, Ph.D. thesis, Norwegian University of Science and Technology (2014).

[24] X. Shan, X. Yuan, H. Chen, Kinetic theory representation of hydrodynamics: a way beyond the navier-stokes equation, Journal of Fluid Mechanics 550 (2006) 413-441.

[25] X. Shan, H. Chen, Simulation of nonideal gases and liquid-gas phase transitions by the lattice boltzmann equation, Phys. Rev. E 49 (1994) 2941-2948. URL http://link.aps.org/doi/10.1103/PhysRevE.49.2941

[26] S. S. Chikatamarla, I. V. Karlin, Entropy and galilean invariance of lattice boltzmann theories Phys. Rev. Lett. 97 (2006) 190601. doi:10.1103/ PhysRevLett.97.190601.

URL https://link.aps.org/doi/10.1103/PhysRevLett.97.190601

[27] S. S. Chikatamarla, S. Ansumali, I. V. Karlin, Entropic lattice boltzmann models for hydrodynamics in three dimensions, Phys. Rev. Lett. 97 (2006) 010201. doi:10.1103/PhysRevLett.97.010201.

URL https://link.aps.org/doi/10.1103/PhysRevLett.97.010201

[28] S. S. Chikatamarla, I. V. Karlin, Lattices for the lattice boltzmann method, Phys. Rev. E 79 (2009) 046701. doi:10.1103/PhysRevE.79.046701.

URL https://link.aps.org/doi/10.1103/PhysRevE.79.046701

[29] Y.-H. Qian, Y. Zhou, Complete galilean-invariant lattice bgk models for the navier-stokes equation, EPL (Europhysics Letters) 42 (4) (1998) 359. URL http://stacks .iop.org/0295-5075/42/i=4/a=359

[30] Y. Wei, H.-S. Dou, Y. Qian, Z. Wang, A novel two-dimensional coupled lattice boltzmann model for incompressible flow in application of turbulence rayleigh-taylor instability, Computers \& Fluids 156 (Supplement C) (2017) 97 - 102, ninth International Conference on Computational Fluid Dynamics (ICCFD9). doi:https://doi.org/10.1016/j.compfluid.2017.07.003.

URL http://www.sciencedirect.com/science/article/pii/ S0045793017302414 
[31] P. A. Skordos, Initial and boundary conditions for the lattice boltzmann method, Phys. Rev. E 48 (1993) 4823-4842. doi:10.1103/PhysRevE.48. 4823.

URL https://link.aps .org/doi/10.1103/PhysRevE.48.4823

[32] J. Buick, C. Greated, Lattice boltzmann modeling of interfacial gravity waves, Physics of Fluids (1994-present) 10 (6) (1998) 1490-1511.

[33] R. Mei, L.-S. Luo, P. Lallemand, D. d'Humières, Consistent initial conditions for lattice boltzmann simulations, Computers \& Fluids 35 (8-9) (2006) 855-862, proceedings of the First International Conference for Mesoscopic Methods in Engineering and Science.

URL http://ww.sciencedirect.com/science/article/pii/ S0045793005001477

[34] X. He, L.-S. Luo, Lattice boltzmann model for the incompressible navierstokes equation, Journal of Statistical Physics 88 (3) (1997) 927-944.

URL http://dx.doi.org/10.1023/B:JOSS.0000015179.12689.e4

[35] R. A. Horn, C. R. Johnson, Norms for Vectors and Matrices, Vol. Ch. 5, Cambridge University Press, Cambridge, England, 1990.

[36] S. Chen, Z. Wang, X. Shan, G. D. Doolen, Lattice boltzmann computational fluid dynamics in three dimensions, Journal of Statistical Physics 68 (3) (1992) 379-400.

URL http://dx.doi.org/10.1007/BF01341754

[37] S. S. Chikatamarla, C. E. Frouzakis, I. V. Karlin, A. G. Tomboulides, K. B. Boulouchos, Lattice boltzmann method for direct numerical simulation of turbulent flows, Journal of Fluid Mechanics 656 (2010) 298-308. doi: $10.1017 / \mathrm{S} 0022112010002740$

\section{AppendixA. Multi-scale analysis}

This section derives the hydrodynamic equations from the kinetic equation 1 and exposes the inherent issues when using incorrect moments. Following the 
literature 44, 5, Eq. 11 is expanded to second-order using a multi-variate Taylor expansion:

$$
\mathrm{D} f_{i}+\frac{1}{2} \mathrm{D}^{2} f_{i} \triangle t \approx \Omega_{i}
$$

where $\mathrm{D}=\partial_{t}+e_{i, \alpha} \partial_{\alpha}$ is the material derivative. Expanding the distribution functions and derivatives:

$$
\begin{gathered}
f_{i}=f_{i, e q}+\epsilon f_{i, 1}+\epsilon^{2} f_{i, 2} \\
\partial_{t}=\epsilon \partial_{t, 1}+\epsilon^{2} \partial_{t, 2} \quad \partial_{\alpha}=\epsilon \partial_{\alpha, 1}
\end{gathered}
$$

the material derivative and collision operator may similarly be expanded:

$$
\begin{gathered}
\Omega_{i}=\epsilon \Omega_{i, 1}+\epsilon^{2} \Omega_{i, 2} \\
\mathrm{D}=\epsilon \mathrm{D}_{1}+\epsilon^{2} \mathrm{D}_{2}
\end{gathered}
$$

where $\Omega_{i, k}=-\frac{f_{i, k}}{\tau}, \mathrm{D}_{1}=\partial_{t, 1}+e_{i, \alpha} \partial_{\alpha, 1}$ and $\mathrm{D}_{2}=\partial_{t, 2}$. Substituting the expansions into Eq. A.1 and gathering all terms of order $\epsilon$ and $\epsilon^{2}$ respectively yields:

$$
\begin{gathered}
\mathrm{D}_{1} f_{i, e q} \approx \Omega_{i, 1} \\
\mathrm{D}_{2} f_{i, e q}-\mathrm{D}_{1}\left[\Delta t\left(\frac{\tau}{\triangle t}-\frac{1}{2}\right) \Omega_{i, 1}\right] \approx \Omega_{i, 2}
\end{gathered}
$$

To reconstruct the macroscopic hydrodynamic equations from these relations requires the specification of the moments of the equilibrium function, i.e. Eq. 6]8. Taking the zeroth moments of the relations:

$$
\begin{gathered}
\partial_{t, 1} \rho+\partial_{\alpha, 1} \rho u_{\alpha}=0 \\
\partial_{t, 2} \rho=0
\end{gathered}
$$

results in the continuity equation in the macroscopic limit:

$$
\partial_{t} \rho+\partial_{\alpha} \rho u_{\alpha}=0
$$


Taking the first moments:

$$
\begin{gathered}
\partial_{t, 1} \rho u_{\alpha}+\partial_{\beta, 1} \pi_{\alpha \beta, e q}=0 \\
\partial_{t, 2} \rho u_{\alpha}+\partial_{\beta, 1} \pi_{\alpha \beta, 1}=0
\end{gathered}
$$

results in the Navier-Stokes equations:

$$
\partial_{t} \rho u_{\alpha}+\partial_{\beta} \pi_{\alpha \beta}=0
$$

where $\pi_{\alpha \beta}=\pi_{\alpha \beta, e q}+\pi_{\alpha \beta, 1}$ is the momentum flux tensor with the equilibrium and non-equilibrium contributions given respectively:

$$
\begin{gathered}
\pi_{\alpha \beta, e q}=\sum_{i} e_{i, \alpha} e_{i, \beta} f_{i, e q} \\
\pi_{\alpha \beta, 1}=-\Delta t\left(\frac{\tau}{\triangle t}-\frac{1}{2}\right) \sum_{i} e_{i, \alpha} e_{i, \beta} \Omega_{i, 1}
\end{gathered}
$$

The equilibrium contribution is directly determined from the requirement of the second moment on the equilibrium distribution in Eq. 7. The nonequilibrium contribution is dependent on the relaxation time $\tau$ and is determined by interaction between particles through the collision process.

Substituting the result for $\Omega_{i, 1}$ from Eq. A.4 yields:

$$
\begin{aligned}
\hat{\pi}_{\alpha \beta, 1} & =\sum_{i} e_{i, \alpha} e_{i, \beta} \mathrm{D}_{1} f_{i, e q} \\
& =\partial_{t, 1} \pi_{\alpha \beta, e q}+\partial_{\gamma, 1} \sum_{i} e_{i, \alpha} e_{i, \beta} e_{i, \gamma} f_{i, e q}
\end{aligned}
$$

where we define $\hat{\pi}_{\alpha \beta, 1} \equiv \frac{\pi_{\alpha \beta, 1}}{-\Delta t\left(\frac{\tau}{\Delta t}-\frac{1}{2}\right)}$ to simplify subsequent calculations. The right-hand side of Eq. A.11 shows that in order to proceed it is required to specify also the third moment of the equilibrium distribution. While not specifically stated in [5, 12], it follows from Eq. 3 that:

$$
\sum_{i} e_{i, \alpha} e_{i, \beta} e_{i, \gamma} f_{i, e q}=\rho c_{s}^{2}\left(u_{\alpha} \delta_{\beta \gamma}+u_{\beta} \delta_{\alpha \gamma}+u_{\gamma} \delta_{\alpha \beta}\right)
$$

It should be noted that Eq. A.12 is in terms of the lattice speed of sound, $c_{s}$, rather than the effective speed of sound $c_{s, e}$. Substituting the third moment into the non-equilibrium momentum flux tensor yields: 


$$
\begin{aligned}
\hat{\pi}_{\alpha \beta, 1}= & \partial_{t, 1}\left(\rho c_{s, e}^{2} \delta_{\alpha \beta}+\rho u_{\alpha} u_{\beta}\right)+\partial_{\gamma, 1}\left(\rho c_{s}^{2} u_{\gamma}\right) \delta_{\alpha \beta} \\
& \quad+\partial_{\alpha, 1}\left(\rho c_{s}^{2} u_{\beta}\right)+\partial_{\beta, 1}\left(\rho c_{s}^{2} u_{\alpha}\right) \\
= & \partial_{t, 1}\left(\rho c_{s, e}^{2} \delta_{\alpha \beta}\right)+\partial_{\gamma, 1}\left(\rho c_{s}^{2} u_{\gamma}\right) \delta_{\alpha \beta} \\
& \quad+\partial_{t, 1}\left(\rho u_{\alpha} u_{\beta}\right)+\partial_{\alpha, 1}\left(\rho c_{s}^{2} u_{\beta}\right)+\partial_{\beta, 1}\left(\rho c_{s}^{2} u_{\alpha}\right)
\end{aligned}
$$

resulting finally in:

$$
\begin{array}{r}
\hat{\pi}_{\alpha \beta, 1}=\partial_{t, 1}\left(\rho c_{s, e}^{2} \delta_{\alpha \beta}\right)+\partial_{\gamma, 1}\left(\rho c_{s}^{2} u_{\gamma}\right) \delta_{\alpha \beta} \\
+\partial_{\alpha, 1}\left(\rho c_{s}^{2} u_{\beta}\right)-u_{\beta} \partial_{\alpha, 1}\left(\rho c_{s, e}^{2}\right) \\
+\partial_{\beta, 1}\left(\rho c_{s}^{2} u_{\alpha}\right)-u_{\alpha} \partial_{\beta, 1}\left(\rho c_{s, e}^{2}\right)
\end{array}
$$

which can be rewritten as:

$$
\hat{\pi}_{\alpha \beta, 1}=\rho c_{s, e}^{2}\left[\partial_{\alpha, 1} u_{\beta}+\partial_{\beta, 1} u_{\alpha}\right]+\hat{\epsilon}_{\alpha \beta}
$$

At this point in Eq. A.14 the top line should be identically zero due to the result of Eq. A.5. Furthermore, the last two lines should simplify to Eq. 11 to recover the appropriate stress tensor. Unfortunately, this is not the case as in general $c_{s, e} \neq c_{s}$ and as a result an error term, $\hat{\epsilon}_{\alpha \beta} \equiv \frac{\epsilon_{\alpha \beta}}{-\Delta t\left(\frac{\tau}{\Delta t}-\frac{1}{2}\right)}$, is introduced:

$$
\hat{\epsilon}_{\alpha \beta}=(1-\gamma) c_{s}^{2}\left[\partial_{\gamma, 1}\left(\rho u_{\gamma}\right) \delta_{\alpha \beta}+\partial_{\alpha, 1}\left(\rho u_{\beta}\right)+\partial_{\beta, 1}\left(\rho u_{\alpha}\right)\right]
$$

where $\gamma=c_{s, e}^{2} / c_{s}^{2}$. It is easily seen that this term is only identically zero when there are no velocity gradients or when $\gamma=1$, i.e. $c_{s, e}=c_{s}$.

To alleviate this issue, the third moment of the equilibrium distribution must likewise be defined in terms of the effective speed of sound:

$$
\sum_{i} e_{i, \alpha} e_{i, \beta} e_{i, \gamma} f_{i, e q}=\rho c_{s, e}^{2}\left(u_{\alpha} \delta_{\beta \gamma}+u_{\beta} \delta_{\alpha \gamma}+u_{\gamma} \delta_{\alpha \beta}\right)
$$

In that case, the result for the non-equilibrium contribution to the momentum flux tensor is:

$$
\hat{\pi}_{\alpha \beta, 1}=-\rho c_{s, e}^{2}\left[\partial_{\alpha, 1} u_{\beta}+\partial_{\beta, 1} u_{\alpha}\right]
$$


This is equivalent to the stress tensor as defined in equation 11 with effective viscosity:

$$
\nu_{e}=c_{s, e}^{2} \triangle t\left(\frac{\tau}{\triangle t}-\frac{1}{2}\right)
$$

which now also depends on the effective speed of sound.

\section{AppendixB. Determining the coefficients of the modified equilibrium}

AppendixB.1. The deficiency of single-speed lattices

For the single-speed D1Q3 lattice, the resulting sets of equations for coefficient $A_{i}$ and $B_{i}$ are:

$$
\begin{gathered}
\frac{2}{3} A_{1}+\frac{1}{3} A_{2 \rightarrow 3}=1 \quad A_{2 \rightarrow 3}=\gamma \\
B_{2 \rightarrow 3}=1 \quad B_{2 \rightarrow 3}=\gamma
\end{gathered}
$$

The coefficients $A_{2 \rightarrow 3}=A_{2}=A_{3}$ and $B_{2 \rightarrow 3}=B_{2}=B_{3}$ have been combined due to the symmetry of the lattice and the assumed symmetry of the coefficients. The solution to the equations for coefficient $B_{i}$ requires $B_{2 \rightarrow 3}=\gamma=1$ which indicates that on a single-speed lattice the speed of sound cannot be tuned without adapting the grid.

The single-speed D2Q9 lattice results in two systems of equations for coefficients $A_{i}$ :

$$
\begin{gathered}
\frac{4}{9} A_{1}+\frac{4}{9} A_{2 \rightarrow 5}+\frac{1}{9} A_{6 \rightarrow 9}=1 \\
\frac{2}{3} A_{2 \rightarrow 5}+\frac{1}{3} A_{6 \rightarrow 9}=\gamma
\end{gathered}
$$

and coefficients $B_{i}$ :

$$
\begin{gathered}
\frac{2}{3} B_{2 \rightarrow 5}+\frac{1}{3} B_{6 \rightarrow 9}=1 \\
2 B_{2 \rightarrow 5}+B_{6 \rightarrow 9}=3 \gamma \\
B_{6 \rightarrow 9}=\gamma
\end{gathered}
$$


As with the $1 \mathrm{D}$ case, the coefficients have been combined such that $A_{2 \rightarrow 5}=$ $A_{2}=A_{3}=A_{4}=A_{5}$ and $B_{2 \rightarrow 5}=B_{2}=B_{3}=B_{4}=B_{5}$, etc. Following the analogy of the single-speed D1Q3 lattice in the 1D implementation, the solution to equations of coefficients $B_{i}$ are $B_{2 \rightarrow 5}=B_{6 \rightarrow 9}=\gamma=1$. Again a single-speed D2Q9 lattice results in the inability to accurately modify the speed of sound.

For both single-speed lattices, values of $\gamma \neq 1$ do not satisfy the equations in AppendixA and we must introduce additional velocity directions as used in multi-speed lattices.

\section{AppendixB.2. Improvement using multi-speed lattices}

For the multi-speed D1Q5 lattice, the resulting sets of equations for coefficient $A_{i}$ are:

$$
\begin{gathered}
\frac{1}{2} A_{1}+\frac{1}{3} A_{2 \rightarrow 3}+\frac{1}{6} A_{4 \rightarrow 5}=1 \\
\frac{1}{3} A_{2 \rightarrow 3}+\frac{2}{3} A_{4 \rightarrow 5}=\gamma
\end{gathered}
$$

and for coefficients $B_{i}$ are:

$$
\begin{aligned}
& \frac{1}{3} B_{2 \rightarrow 3}+\frac{2}{3} B_{4 \rightarrow 5}=1 \\
& \frac{1}{3} B_{2 \rightarrow 3}+\frac{8}{3} B_{4 \rightarrow 5}=3 \gamma
\end{aligned}
$$

The set of equations form a linearly independent system of four equations for a total of six unknowns (e.g. $A_{1}, A_{2 \rightarrow 3}, A_{4 \rightarrow 5}, B_{2 \rightarrow 3}, B_{4 \rightarrow 5}$ and $c_{s, e}$ ) requiring two additional relations. By setting the relation $B_{4 \rightarrow 5}=r B_{2 \rightarrow 3}$, solutions for $B_{2 \rightarrow 3}, B_{4 \rightarrow 5}$ and $c_{s, e}$ are readily found. Finally, for coefficients $A_{i}$, the relation $A_{4 \rightarrow 5}=r A_{2 \rightarrow 3}$ is chosen from which follows coefficients $A_{1}, A_{2 \rightarrow 3}$ and $A_{4 \rightarrow 5}$. Note that any permutation of arbitrary relations between the coefficients for $A_{i}$ and $B_{i}$ will lead to a solution for the coefficients. The solution to the set of equations is:

$$
\begin{gathered}
A_{1}=\frac{16 r^{2}+7 r+4}{12 r^{2}+12 r+3} \quad A_{2 \rightarrow 3}=\frac{8 r+1}{(2 r+1)^{2}} \\
B_{1}=0 \quad B_{2 \rightarrow 3}=\frac{3}{2 r+1} \\
A_{4 \rightarrow 5}=r A_{2 \rightarrow 3} \quad B_{4 \rightarrow 5}=r B_{2 \rightarrow 3}
\end{gathered}
$$


with a speed of sound, $c_{s, e}$, defined through the ratio $\gamma$ :

$$
\gamma=\frac{c_{s, e}^{2}}{c_{s}^{2}}=\frac{1}{3} \frac{8 r+1}{2 r+1}
$$

All coefficients $A_{i}=B_{i}=\gamma=1$ when $r=1$ corresponding to the conventional case as expected.

Contrary to the single-speed lattice, the multi-speed lattice allows the modification of the speed of sound at the cost of a more computationally expensive scheme.

For the multi-speed D2Q13 lattice, the resulting sets of equations for coefficient $A_{i}$ are:

$$
\begin{gathered}
\frac{3}{8} A_{1}+\frac{1}{3} A_{2 \rightarrow 5}+\frac{1}{4} A_{6 \rightarrow 9}+\frac{1}{24} A_{10 \rightarrow 13}=1 \\
\frac{1}{3} A_{2 \rightarrow 5}+\frac{1}{2} A_{6 \rightarrow 9}+\frac{1}{6} A_{10 \rightarrow 13}=\gamma
\end{gathered}
$$

and for coefficients $B_{i}$ are:

$$
\begin{gathered}
\frac{1}{3} B_{2 \rightarrow 5}+\frac{1}{2} B_{6 \rightarrow 9}+\frac{1}{6} B_{10 \rightarrow 13}=1 \\
\frac{2}{3} B_{2 \rightarrow 5}+B_{6 \rightarrow 9}+\frac{4}{3} B_{10 \rightarrow 13}=3 \gamma \\
B_{6 \rightarrow 9}=\gamma
\end{gathered}
$$

Analogous to the 1D case, the set of equations form a linearly independent system of five equations for a total of eight unknowns (e.g. $A_{1}, A_{2 \rightarrow 5}, A_{6 \rightarrow 9}$, $A_{10 \rightarrow 13}, B_{2 \rightarrow 5}, B_{6 \rightarrow 9}, B_{10 \rightarrow 13}$ and $\left.c_{s, e}\right)$ requiring three additional relations. By setting the relation $B_{10 \rightarrow 13}=r B_{6 \rightarrow 9}$, solutions for $B_{2 \rightarrow 3}, B_{4 \rightarrow 5}$ and $c_{s, e}$ are readily found. For the coefficients $A_{i}$, the relations $A_{6 \rightarrow 9}=r A_{2 \rightarrow 5}$ and $A_{10 \rightarrow 13}=r A_{6 \rightarrow 9}$ are chosen from which follow the coefficients $A_{1}, A_{2 \rightarrow 3}$ and $A_{4 \rightarrow 5}$. Note that any permutation of arbitrary relations between the coefficients for $A_{i}$ and $B_{i}$ will lead to a solution for the coefficients. The solution to the set of equations is: 


$$
\begin{gathered}
A_{1}=\frac{2 \gamma}{3}\left[\frac{4+8 r-3 r^{2}}{2+3 r+r^{2}}\right] \quad A_{2 \rightarrow 5}=\frac{12}{6+7 r-r^{3}} \\
B_{1}=0 \quad B_{2 \rightarrow 5}=\frac{6-4 r}{3-r} \quad B_{6 \rightarrow 9}=\gamma \\
A_{6 \rightarrow 9}=r A_{2 \rightarrow 5} \quad A_{10 \rightarrow 13}=r A_{6 \rightarrow 9} \quad B_{10 \rightarrow 13}=r B_{6 \rightarrow 9}
\end{gathered}
$$

with a speed of sound defined through the ratio $\gamma$ :

$$
\gamma=\frac{c_{s, e}^{2}}{c_{s}^{2}}=\frac{2}{3-r}
$$

Again, all coefficients $A_{i}=B_{i}=\gamma=1$ when $r=1$ corresponding to the conventional case as expected. 